\title{
New groups of planetary nebulae with peculiar dust chemistry towards the Galactic bulge ${ }^{\star}$
}

\author{
S. K. Górny ${ }^{1}$, J. V. Perea-Calderón ${ }^{2}$, D. A. García-Hernández ${ }^{3}$, P. García-Lario ${ }^{4}$, and R. Szczerba ${ }^{1}$ \\ 1 N. Copernicus Astronomical Center, Rabiańska 8, 87-100 Toruń, Poland \\ e-mail: skg@ncac. torun.pl \\ 2 European Space Astronomy Centre, INSA S.A. PO Box 50727, 28080 Madrid, Spain \\ Instituto de Astrofísica de Canarias, C/ Via Láctea s/n, 38200 La Laguna, Spain \\ ${ }^{4}$ Herschel Science Centre, European Space Astronomy Centre, Research and Scientific Support Department of ESA, \\ Villafranca del Castillo, PO Box - Apdo. 50727, 28080 Madrid, Spain
}

Received 29 July 2009 / Accepted 15 January 2010

\section{ABSTRACT}

\begin{abstract}
Aims. We investigate Galactic bulge planetary nebulae without emission-line central stars for which peculiar infrared spectra have been obtained with the Spitzer Space Telescope, including the simultaneous signs of oxygen and carbon based dust. Three separate subgroups can be defined characterized by the different chemical composition of the dust and the presence of crystalline and amorphous silicates.

Methods. We use literature data to analyze the different nebular properties and deduce both the evolutionary status and the origin of these three groups. In particular, we check whether there are signs of evolutionary links between dual-dust chemistry planetary nebulae without detected emission-line central stars and those with emission-line stars.

Results. Our primary finding is that the classification based on the dust properties is reflected in the more general properties of these planetary nebulae. However, some observed properties are difficult to relate to the common view of planetary nebulae. In particular, it is challenging to interpret the peculiar gas chemical composition of many analyzed objects in the standard picture of the evolution of planetary nebulae progenitors. We confirm that the dual-dust chemistry phenomenon is not limited to planetary nebulae with emissionline central stars.

Conclusions. Our results clearly indicate that there is no unique road to the formation of planetary nebulae even in a homogeneous environment such as the Galactic bulge. The evolution of a single asymptotic giant branch star may lead to the formation of different types of planetary nebulae. In addition, the evolution in a close binary system should sometimes also be considered.
\end{abstract}

Key words. planetary nebulae: general - Galaxy: bulge - infrared: stars - stars: Wolf-Rayet

\section{Introduction}

After the completion of hydrogen and helium burning in their cores, low- to intermediate-mass stars $\left(0.8 \leq M \leq 8 M_{\odot}\right)$ evolve towards the asymptotic giant branch (AGB; e.g., Herwig 2005) and then pass through the planetary nebula (PN, plural $\mathrm{PNe}$ ) phase before ending their lives as white dwarfs. At the tip of the AGB phase, these stars experience strong mass loss that efficiently enriches the surrounding interstellar medium with huge amounts of gas and dust. Stars leave the AGB when the strong mass loss stops and then the future central star (CS, plural CSs) rapidly evolves towards hotter effective temperatures in the Hertzsprung-Russell diagram. Thus, when the ionization of the ejected gas takes place, a new PN is formed. However, in most cases the total amount of ionized gas is very small compared to the total mass previously ejected. An important fraction of this material remains neutral in the form of dust grains, molecules, or atoms, which can be easily detected in the infrared domain. Thanks to the analysis of the features observed by the Infrared Space Observatory (ISO) in the spectra of $\mathrm{PNe}$, it was possible to

* Based on observations made with the Spitzer Space Telescope, which is operated by the Jet Propulsion Laboratory, California Institute of Technology, under NASA contract 1407. confirm the presence of large amounts of dust grains around $\mathrm{PNe}$ as well as the dominant dust chemistry (C-rich versus O-rich). Features at 3.3, 6.2, "7.7", 8.6, and $11.3 \mu \mathrm{m}$ attributed to polycyclic aromatic hydrocarbons (PAHs) are common in C-rich PNe (e.g., García-Lario et al. 1999, and references therein) while strong features attributed to crystalline silicates (e.g., those centered on 23.5, 27.5 and $33.8 \mu \mathrm{m}$ ) are usually found in O-rich PNe (e.g., Molster et al. 2002).

A few Galactic disk PNe exhibited a remarkable dual-dust (C-rich and O-rich) chemistry showing both PAH and crystalline silicate features in ISO spectra (Waters et al. 1998a,b; Cohen et al. 1999, 2002). The fact that this was an infrequent phenomenon may be due to the instruments used, which in many cases may have been unable to detect crystalline silicates. For example, the Spitzer Space Telescope (Spitzer, Werner et al. 2004), detected crystalline silicates in 10 post-AGB sources (Cerrigone et al. 2009) while after completion of the ISO mission only 2 such sources were known (Szczerba et al. 2003).

The mixed-chemistry PNe discovered by ISO pertain to the class of objects with C-rich Wolf-Rayet type nuclei (the so-called [WR] PNe), which usually show a lack of hydrogen in their atmospheres. These atmospheres are instead mostly 
composed of helium, carbon, and oxygen and the CSs show intense mass-loss (e.g., Crowther 2008).

The evolution of an AGB star with a stellar or substellar companion that undergoes the common-envelope phase is another possible way of creating a PN. Some authors argue that a companion object is often mandatory for a planetary nebula to be created (see in de Marco 2009, and references therein). We noted that some hypotheses compiled to explain the simultaneous presence of carbon and oxygen dust also require a binary system.

The Galactic bulge is characterized by an old population of mostly low-mass stars (Zoccali et al. 2003; but see also Uttenthaler et al. 2007, and references therein). It is also known that the abundances of PNe in the Galactic bulge (GBPNe) differ from those located in the Galactic disk as they have higher metallicities and lower C/O ratios (e.g., Wang \& Liu 2007). The differences in metallicity seems to play a dominant role in the chemical evolution of low- to intermediate-mass stars (e.g., García-Hernández et al. 2007; Stanghellini et al. 2007; Chiappini et al. 2009). Thus, studding the Galactic bulge enables us to investigate the stellar evolution of low- and intermediate-mass stars in higher metallicity environments and at the same time an insight into the chemical evolution and formation of our Milky Way.

Gutenkunst et al. (2008) analyzed Spitzer spectra acquired using the Infrared Spectrograph (IRS, Houck et al. 2004) of $11 \mathrm{PNe}$ towards the Galactic bulge and inferred dual-dust chemistry in 6 of them. They suggested that the high percentage of dual-dust chemistry sources is unsurprising because the fraction of [WR] PNe is significantly higher in the bulge than in the Galactic disk. However, as we checked, only one of their dual-dust chemistry sources have the wind characteristics of [WR] type CS and the higher proportion of genuine [WR] PNe in the Galactic bulge is not confirmed (Górny et al. 2009). Perea-Calderón et al. (2009) found that dual-dust chemistry is truly widespread among GBPNe. They analyzed a larger sample of 26 GBPNe observed with Spitzer/IRS among which 21 exhibit dual-dust chemistry. Perea-Calderón et al. (2009) observations shown that the simultaneous presence of oxygen and carbon-rich dust features in the infrared spectra of [WR] PNe is not restricted to objects with late/cool [WC] class stars. In addition, dual-dust chemistry was seen in all observed PNe with WEL stars (weak emission-line stars, Tylenda et al. 1993) as well as members of recently discovered VL group (low ionization $\mathrm{PNe}$ around stars with very late [WC 11]-like spectra, Górny et al. 2009). Surprisingly, Perea-Calderón et al. (2009) found dual-dust chemistry also in some PNe without detected emission-line CSs.

Another interesting property of the $\mathrm{PNe}$ observed by Perea-Calderón et al. (2009) was the amorphous silicate emission at $10 \mu \mathrm{m}$, which was detected in four dual-dust chemistry GBPNe and in most of the O-rich PNe that they observed. Note that before Spitzer there was known only one such PN, namely SwSt 1 (e.g., Szczerba et al. 2001), belonging to the [WR] PNe. In contrast, the $10 \mu \mathrm{m}$ feature objects found by Perea-Calderón et al. (2009), neither belong to this group nor exhibit stellar emission lines.

In this work, we analyze the multiple properties of PNe without emission-line CSs that are found to have peculiar infrared spectra acquired with Spitzer/IRS. The paper is organized as follows. In Sects. 2 and 3 we describe our working sample and the main properties of their infrared spectra, respectively. We analyze the nebular properties and evolutionary status of these $\mathrm{PNe}$
Table 1. List of analyzed PNe.

\begin{tabular}{lllllll}
\hline \hline PN G & Name & Pop. & Ref. & \multicolumn{3}{c}{ IR spectra } \\
& & & & PAH & SiO cr. SiO am. \\
\hline DC $_{\mathrm{cr}}$ : & & & & & & \\
$000.1+04.3$ & H 1-16 & b & P & + & + & - \\
$007.2+01.8$ & Hb 6 & d & P & + & + & - \\
$354.2+04.3$ & M 2-10 & b & G & + & + & - \\
$358.7-05.2$ & H 1-50 & b & P & + & + & - \\
$358.9+03.2$ & H 1-20 & b & G & + & + & - \\
DC $\mathrm{a}+\mathrm{cr}:$ & & & & & & \\
$354.5+03.3$ & Th 3-4 & b & P & + & + & + \\
$356.9+04.4$ & M 3-38 & b & P & + & + & + \\
$358.2+04.2$ & M 3-8 & b & P & + & + & + \\
$359.7-02.6$ & H 1-40 & b & P & + & + & + \\
OC $\mathrm{a}+\mathrm{cr}:$ & & & & & & + \\
$002.2-02.7$ & M 2-23 & b & P & - & + & + \\
$008.2+06.8$ & He 2-260 & d & P & - & + & + \\
$355.6-02.7$ & H 1-32 & b & P & - & + & + \\
$355.7-03.5$ & H 1-35 & b & P & - & + & + \\
\hline
\end{tabular}

Notes. The Galactic bulge (b) or disk (d) association of the object is marked in Col. 3. The reference to original observers is given in Col. 4 . Objects are divided into three groups according to their infrared properties as marked in Cols. 5-7 (see description in Sect. 3).

References. G - Gutenkunst et al. (2008); P - Perea-Calderón et al. (2009).

in Sect. 4. Finally, in Sect. 5 we discuss the results obtained. Our concluding remarks are given in Sect. 6.

\section{Sample selection}

We analyze the various properties of PNe without emission-line CSs observed with Spitzer/IRS and exhibiting signs of dual-dust chemistry and/or $10 \mu \mathrm{m}$ emission band of amorphous silicates. These PNe are listed in Table 1 along with a short description of their infrared features. Most of them were observed by Perea-Calderón et al. (2009), while two come from Gutenkunst et al. (2008).

All of the selected PNe were included in the analyses of Górny et al. (2009) but none were attributed to either WEL, VL, or [WR]-type groups of GBPNe. The absence of emission-line CSs was perceived in these PNe by studying high quality optical spectra acquired at 2 and 4-m telescopes by Cuisinier et al. (2000), Escudero \& Costa (2001), Escudero et al. (2004), Górny et al. (2004), and new observations in Górny et al. (2009), or by checking the list of the observed lines in Wang \& Liu (2007). The searched stellar emission lines were the same as in Górny et al. (2004). In none of the thirteen PNe presented in Table 1 did we notice any characteristics of emission-line CSs during additional direct re-inspection of the spectra.

The objects for which the CS was of unknown type are not considered until their spectral classification is established. The PNe with dual-dust chemistry but unknown spectral type of the CS are H 2-20 and M 2-5 observed by Gutenkunst et al. (2008) ${ }^{1}$ and H 1-62 by Perea-Calderón et al. (2009). The spectral type of the CS is also unknown for three O-rich PNe with the detected $10 \mu \mathrm{m}$ emission feature, i.e., He 3-1357, Cn 1-3 and IC 4732 listed by Perea-Calderón et al. (2009).

\footnotetext{
1 The remaining dual-dust chemistry $\mathrm{PNe}$ on their list belong to [WR] type (M 2-31) and WEL PNe (H 2-11).
} 
Eleven of the PNe listed in Table 1 have a high probability of physically belonging to the Milky Way bulge because they satisfy the standard criteria (Stasińska \& Tylenda 1994), namely: a) they are located within 10 degrees of the center of the Galaxy, b) have diameters smaller than $20^{\prime \prime}$, and c) the known radio fluxes at $5 \mathrm{GHz}$ are lower than $100 \mathrm{mJy}$. The two remaining objects are either too bright in the radio domain ( $\mathrm{Hb} 6)$ or marginally outside the 10 degree angular distance (He 2-260) and therefore regarded as possible Galactic disk members.

The 13 objects collected in Table 1 are compared in this paper with the remainder of the total of 180 GBPNe analyzed in Górny et al. (2009). Among this large reference group, there are 119 PNe without emission-line CSs (which we refer to as normal PNe throughout this paper), $25 \mathrm{WEL}, 14 \mathrm{VL}$, and 9 [WR] PNe. Some of the PNe from the latter groups were also observed with Spitzer/IRS by either the Perea-Calderón or Gutenkunst teams. This includes 5 WEL, 4 [WR], and 3 VL PNe.

In this paper, we compare the objects investigated here to both normal PNe and PNe with emission-line CSs since it can not be excluded that the latter, in particular [WR] $\mathrm{PNe}^{2}$, have at times weaker stellar winds and emission lines from CSs are not observed. It has to be checked if this is not the case of some of the PNe from Table 1. On the other hand, the stellar emission lines of WEL and VL PNe are not very strong and may escape unnoticed in spectra of lower signal to noise. Therefore, to confirm that indeed dual-dust chemistry phenomenon also occurs in PNe without emission-line CSs we compare their properties with those of [WR], WEL, and VL PNe.

Among the largest group of 119 normal bulge PNe that we use as a reference sample only four have been observed with Spitzer/IRS. This is a clear result of the selection effect since the targets for observations were chosen by Perea-Calderón et al. (2009) based on high quality IRAS fluxes at 12,25 , and $60 \mu \mathrm{m}$. In particular, the requirement of reliable measurements at $12 \mu \mathrm{m}$ is fulfilled by only 15 normal PNe of 64 with IRAS data available. This means that no prominent emission features around $12 \mu \mathrm{m}$ should be expected in normal PNe. Even in the case of the four objects observed by Spitzer, only crystalline silicates are present in their spectra and both PAH and amorphous silicates seem to be absent ${ }^{3}$. Unfortunately, the existing optical spectra of these four PNe are at the same time of low quality implying e.g., that the nature of their CSs is uncertain and many parameters cannot be reliably determined.

We add to the discussion the analysis of two PNe from the Galactic disk population. One is the [WR]-type SwSt 1 mentioned already in the introduction and the other is IC 4776 that has a WEL CS and was observed by Perea-Calderón et al. (2009). Both these objects exhibit the dual-dust chemistry signatures typical of PNe with emission-line CSs but additionally also the $10 \mu \mathrm{m}$ amorphous silicate feature.

\section{Infrared spectra}

The infrared spectra of the GBPNe without emission-line CSs selected for this work were acquired by Perea-Calderón et al. (2009) and Gutenkunst et al. (2008). The description of the

\footnotetext{
2 In the Galactic bulge, only [WR] PNe with CSs of intermediate [WC] spectral classes are known, in contrast to the situation in the Galactic disk (see e.g., Górny et al. 2001).

3 Among these PNe, object M3-13 has exceptional Spitzer spectra with unidentified broad emission features at shorter wavelengths, see Fig. B4 of Perea-Calderón et al. (2009).
}

Spitzer/IRS data reduction process is not repeated here and we refer the reader to these two references for details.

After a short inspection of the Spitzer/IRS spectra, we find that among PNe without emission-line CSs, there are three clearly different types of objects. The examples are presented in Fig. 1. It can be seen that $\mathrm{H} \mathrm{1-16}$ and Th 3-4 PNe have mixed chemistry where both PAHs and crystalline silicates features are detected. For Th 3-4 we also detect the amorphous silicate emission feature at $10 \mu \mathrm{m}$. On the other hand, M2-23 is clearly O-rich (there is a lack of carbon-based dust features such as PAHs), exhibiting the amorphous silicate feature at $10 \mu \mathrm{m}$ and weak crystalline silicate features at longer wavelengths. Finally, we note that M2-23 contains a circumstellar dust envelope, which is hotter than in $\mathrm{H} \mathrm{1-16}$ and Th 3-4 as can be inferred from the shape of continuum emission in the spectrum.

Based on the examples from Fig. 1, we can describe the spectra of all PNe analyzed in this work and divide them into different subgroups (Table 1). Each group has some similarity with one of the other groups and clear dissimilarity with another. The first group of 5 PNe listed at the top of Table 1 is characterized by the simultaneous presence of both carbon-based dust (PAH features at 6.2, "7.7", 8.6, and $11.3 \mu \mathrm{m}$ ) and oxygen-based dust (crystalline silicate features at 23.5, 27.5, and $33.8 \mu \mathrm{m}$ ). We refer to this group as $\mathrm{DC}_{\mathrm{cr}}$ (dual-dust chemistry with silicates only in crystalline form) subsample throughout the rest of this paper. The second group in Table 1 has 4 members and is also characterized by the simultaneous presence of oxygen and carbon dust but at the same time there are also signs of amorphous silicate features at about $10 \mu \mathrm{m}$. We therefore refer to this group as $\mathrm{DC}_{\mathrm{a}+\mathrm{cr}}$ (dual-dust chemistry with silicates in amorphous and crystalline form). Finally, 4 PNe in Table 1 exhibit only oxygen dust features, which however include the uncommon $10 \mu \mathrm{m}$ amorphous silicate feature. This last group is called $\mathrm{OC}_{\mathrm{a}+\mathrm{cr}}$ (oxygen-dust chemistry with amorphous and crystalline forms) subsample.

Detailed comparison and analysis of dust features observed with Spitzer/IRS in GBPNe will be presented in the forthcoming paper (Szczerba et al. in prep.)

\section{Nebular properties and evolutionary status}

We analyze different nebular properties that can shed the light on the three groups of bulge PNe and allow us to infer their evolutionary status and origin. Since some of them (in particular $\mathrm{DC}_{\mathrm{cr}}$ ) have infrared spectra resembling those of PNe with emission-line CSs, we also check whether any other properties would associate them with such objects.

In Table 2, we report the basic nebular parameters of the investigated PNe. Most of them were adopted from Gorny et al. (2009), recomputed using the methods and formulae from Stasińska \& Szczerba (1999), or adopted from other literature sources.

\subsection{Bulge location}

As mentioned in Sect. 2, most of the PNe analyzed here are not only simply observed towards the center of the Milky Way but most probably physically pertain to the Galactic bulge. In particular, they are located less than $10^{\circ}$ from the center of the Galaxy. Figure 2 presents the radial velocities $V_{\mathrm{lsr}}$ of bulge PNe corrected 

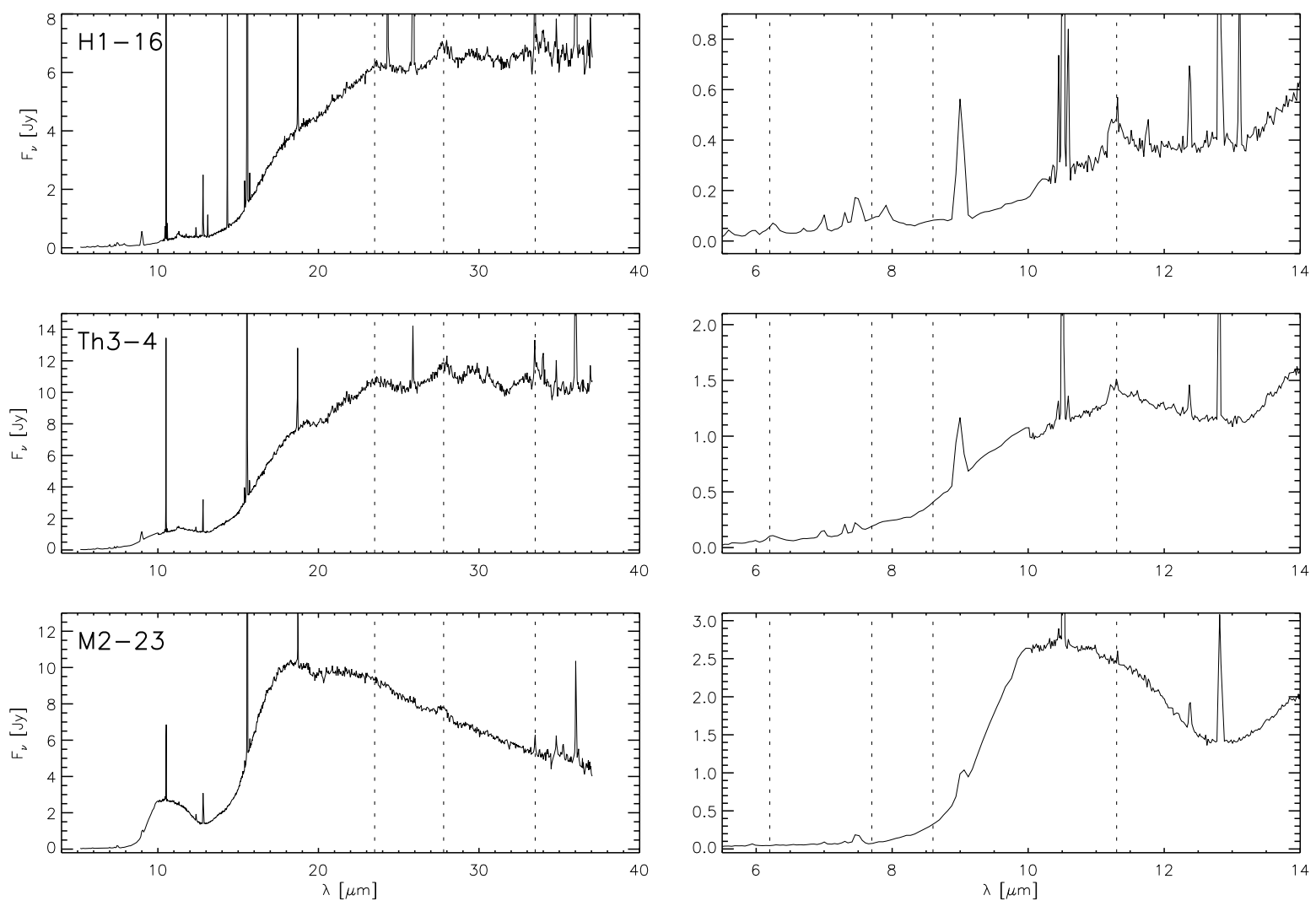

Fig. 1. Examples of Spitzer/IRS spectra of PNe with three different types of dust composition: H 1-16 of DC cr $_{\text {type }}$ at the top, Th 3-4 of DC a+cr $_{\text {in }}$ the middle, and M 2-23 of $\mathrm{OC}_{\mathrm{a}+\mathrm{cr}}$ type at the bottom. Dotted vertical lines indicate the positions of crystalline silicate emission features at 23.5, 27.5 , and $33.8 \mu \mathrm{m}$ (left panels) and typical PAH features at 6.2, "7.7", 8.6, and $11.3 \mu \mathrm{m}$ (right panels). The $10 \mu \mathrm{m}$ amorphous silicate feature is seen in the middle and bottom spectra.

for solar motion ${ }^{4}$ as a function of Galactic longitude coordinate $l$. Although the samples of $\mathrm{DC}_{\mathrm{cr}}, \mathrm{DC}_{\mathrm{a}+\mathrm{cr}}$, and $\mathrm{OC}_{\mathrm{a}+\mathrm{cr}}$ are not numerous, we can state that members of each of them are found at different longitudes within the bulge and no grouping at special locations can be distinguished ${ }^{5}$.

As can be noted in Fig. 2, the velocities of Galactic bulge $\mathrm{DC}_{\mathrm{cr}}, \mathrm{DC}_{\mathrm{a}+\mathrm{cr}}$, and $\mathrm{OC}_{\mathrm{a}+\mathrm{cr}}$ objects (large filled symbols) are usually large or very large and therefore their kinematic properties are typical of PNe physically related to the bulge system (compare with Fig. 13 of Górny et al. (2004)). On the other hand, the two objects already assigned to Galactic disk (Hb 6 and He 2-260) have small $V_{\text {lsr }}$ velocities that are characteristic of that PNe population.

For the other Galactic coordinate, the latitude $b$, the bulge PNe analyzed here show nothing peculiar in their distribution. They are found at $b$ smaller than about $5^{\circ}$, but, as for all the other known PNe, avoid latitudes below $2^{\circ}$. This is simply because of the interstellar dust prohibiting their discovery. However, in Fig. 3 we plot the bulge PNe in $b$ coordinates versus logarithmic extinction parameter at $\mathrm{H} \beta\left(C_{\text {opt }}\right)$ and some display a very

\footnotetext{
4 The radial velocities used in this paper have been taken from Durand et al. (1998) and corrected to the local standard of rest using the formulae of Beaulieu et al. (2000).

5 Recently Górny et al. (2009) noticed a difference in locations between bulge [WR], VL, and WEL PNe with the last two groups typically being located at longitudes less than 4.5 from the center whereas bulge [WR] PNe are often found at greater longitudes. Obviously, the 4.5 longitude does not seem to be important for any of the subsamples investigated here.
}

interesting property: the members of $\mathrm{DC}_{\mathrm{a}+\mathrm{cr}}$ (in particular Th 3-4 and $\mathrm{H} \mathrm{1-40)}$ have much greater extinction than expected of typical PNe located at their latitudes off the Galactic plane.

The extinction plotted in Fig. 3 (see also Col. 2 of Table 2) were derived from the ratios of hydrogen lines in optical spectra. This was accomplished mainly by comparing the observed Balmer $\mathrm{H} \alpha / \mathrm{H} \beta$ ratio with its theoretically expected value, although sometimes $\mathrm{H} \alpha / \mathrm{H} \gamma$ had to be used (see details in Górny et al. 2009). We checked that when extinction can be derived from both of these ratios the agreement is usually very good.

The value of PNe extinction may be above average when the matter along the line of sight has a different blocking properties or if the object is simply located behind a larger amount of interstellar dust. This is the most straightforward explanation, although the chances of finding all four $\mathrm{DC}_{\mathrm{a}+\mathrm{cr}}$ with these conditions can be evaluated at only a few percent. Therefore at least partly the excessive extinction towards $\mathrm{DC}_{\mathrm{a}+\mathrm{cr}}$ could also be attributed to some specific properties of their own dust or the dust nearby around them. In that way, $\mathrm{DC}_{\mathrm{a}+\mathrm{cr}}$ would be interesting candidates for studying the internal extinction of $\mathrm{PNe}$.

\subsection{Diameters and densities}

In Fig. 4, the reddening-corrected fluxes of the hydrogen $\mathrm{H} \beta$ line of GBPNe are presented as a function of apparent angular diameters (Col. 6 in Table 2). As a reference we overplotted the theoretical tracks for PNe at a distance of $8.5 \mathrm{kpc}$ with CSs of three different masses $0.57,0.60$, and $0.64 M_{\odot}$, evolving according to the predictions of Blöcker (1995) and assuming they radiate 
Table 2. Observational data for analyzed planetary nebulae.

\begin{tabular}{lccccccccccc}
\hline \hline Name & $\mathrm{C}_{\mathrm{opt}}$ & $\mathrm{O}^{++} / \mathrm{O}$ & $\mathrm{He}^{++} / \mathrm{He}$ & $\begin{array}{c}\log n_{\mathrm{e}} \\
{\left[\mathrm{cm}^{-3}\right]}\end{array}$ & $\begin{array}{c}\theta \\
{\left[{ }^{\prime \prime}\right]}\end{array}$ & $\begin{array}{c}V_{\text {exp }} \\
{\left[\mathrm{km} \mathrm{s}^{-1}\right]}\end{array}$ & $\begin{array}{c}t_{\text {kin }} \\
{[\text { year }]}\end{array}$ & $\begin{array}{c}\log \mathrm{S}_{\mathrm{H} \beta} \\
{\left[\mathrm{erg} \mathrm{cm}^{-2} \mathrm{~s}^{-1} \mathrm{sr}^{-1}\right]}\end{array}$ & $\begin{array}{c}\log \mathrm{T}_{\text {Zan }} \\
{[\mathrm{K}]}\end{array}$ & $\begin{array}{c}M_{\text {gas }} \\
{\left[M_{\odot}\right]}\end{array}$ & $\begin{array}{c}M_{\text {dust }} \\
{\left[10^{-3} M_{\odot}\right]}\end{array}$ \\
\hline H 1-16 & 2.50 & 0.880 & 0.097 & 3.77 & 2.0 & $20^{\mathrm{R}}$ & $2000^{r}$ & -0.83 & - & 0.152 & 0.38 \\
Hb 6 & 2.10 & 0.901 & 0.115 & 3.53 & 6.0 & $20^{\mathrm{RRA}}$ & $500^{s}$ & -0.95 & $>4.48$ & 0.2 & 1.20 \\
M 2-10 & 1.32 & 0.625 & - & 3.10 & 4.2 & & & -2.00 & - & 0.208 & 1.00 \\
H 1-50 & 0.68 & 0.959 & 0.093 & 3.69 & 1.4 & $23^{\mathrm{GZ}, \mathrm{R}}$ & 1300 & -0.62 & 4.77 & 0.148 & 0.18 \\
H 1-20 & 2.31 & 0.896 & 0.013 & 3.57 & 3.3 & $18^{\mathrm{R}}$ & $3800^{r}$ & -1.30 & - & 0.223 & 0.68 \\
Th 3-4 & 2.83 & 0.946 & 0.078 & 4.20 & 1.5 & & & -0.60 & - & 0.055 & 0.49 \\
M 3-38 & 2.06 & 0.968 & 0.238 & 3.49 & 1.8 & $15^{\mathrm{R}}$ & $2400^{r}$ & -1.05 & - & 0.143 & 1.04 \\
M 3-8 & 1.93 & 0.860 & - & 3.73 & 3.2 & & & -1.48 & 4.50 & 0.095 & 0.53 \\
H 1-40 & 2.52 & 0.939 & - & 4.26 & 3.0 & $20^{\mathrm{GZ}, \mathrm{R}}$ & 1000 & -1.23 & - & 0.044 & 0.66 \\
M 2-23 & 1.28 & 0.924 & - & 4.00 & 1.0 & $14^{\mathrm{R}}$ & $1500^{r}$ & -0.15 & 4.73 & 0.108 & 0.02 \\
He 2-260 & 0.76 & 0.012 & - & 4.16 & 1.0 & $17^{\mathrm{G}}$ & 3500 & -0.65 & 4.41 & 0.2 & 0.74 \\
H 1-32 & 1.63 & 0.787 & - & 3.74 & 1.0 & $11^{\mathrm{R}}$ & $1900^{r}$ & -1.37 & - & 0.012 & 0.07 \\
H 1-35 & 1.33 & 0.525 & 0.002 & 4.26 & 1.5 & $10^{\mathrm{GZ}}$ & 3100 & -0.26 & 4.65 & 0.103 & 0.20 \\
\hline
\end{tabular}

Notes. The reported quantities are: logarithmic extinction at $\mathrm{H} \beta(\mathrm{Col} .2)$; ionization parameters $\mathrm{O}^{++} /\left(\mathrm{O}^{+}+\mathrm{O}^{++}\right)$and $\mathrm{He}^{++} /\left(\mathrm{He}^{+}+\mathrm{He}^{++}\right)(\mathrm{Cols} .3$ and 4); electron density from [S II] $16717 / 6731$ line ratio (Col. 5); nebular diameter (Col. 6); nebular expansion velocity (Col. 7); kinematic age (Col. 8); H $\beta$ surface brightness (Col.9); stellar temperature from Zanstra hydrogen method (Col. 10); mass of ionized nebular gas (Col. 11) and mass of dust (Col. 12).

Kinematic ages $t_{\text {kin }}$ are from Gesicki \& Zijlstra (2000) and Gesicki et al. (2006) or calculated assuming distance $8.5 \mathrm{kpc}(r)$ or from Shklovski method $(s)$.

References. RRA - Robinson et al. (1982), GZ - Gesicki \& Zijlstra (2000), G - Gesicki et al. (2006), R - Richer et al. (2008).

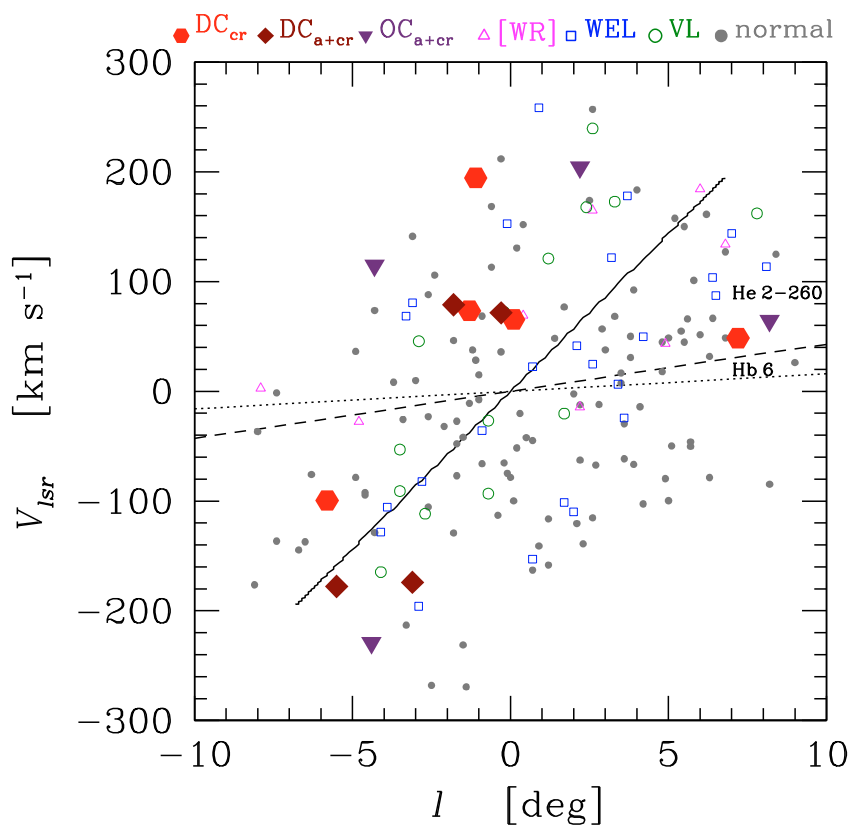

Fig. 2. Radial velocity versus Galactic longitude coordinate of the investigated PNe. The big filled symbols mark: $\mathrm{DC}_{\mathrm{cr}}$ as red hexagons, $\mathrm{DC}_{\mathrm{a}+\mathrm{cr}}$ brown diamonds, and $\mathrm{OC}_{\mathrm{a}+\mathrm{cr}}$ as violet reversed triangles. Galactic bulge PNe with emission-line CSs are marked with smaller open symbols: [WR] as pink triangles, WEL as blue squares, and VL as green circles. Normal Galactic bulge PNe are presented with small dark grey dots. Rotation curves for PNe at 1, 4, and 6 kpc circular orbits assuming Galactocentric rotation velocities of $220 \mathrm{~km} \mathrm{~s}^{-1}$ are plotted with solid, dashed, and dotted lines respectively.

like a black body. The surrounding nebula were described by a simple model of uniformly filled sphere with total gas mass of $0.2 M_{\odot}$, filling factor $\epsilon=0.75$, and expansion at the constant velocity of $20 \mathrm{~km} \mathrm{~s}^{-1}$. As can be seen in this figure, the $\mathrm{DC}_{\mathrm{cr}}$ and $\mathrm{DC}_{\mathrm{a}+\mathrm{cr}} \mathrm{PNe}$ occupy a rather restricted region of the plot. Their $\mathrm{H} \beta$ fluxes are typically 2 times lower than those of the majority

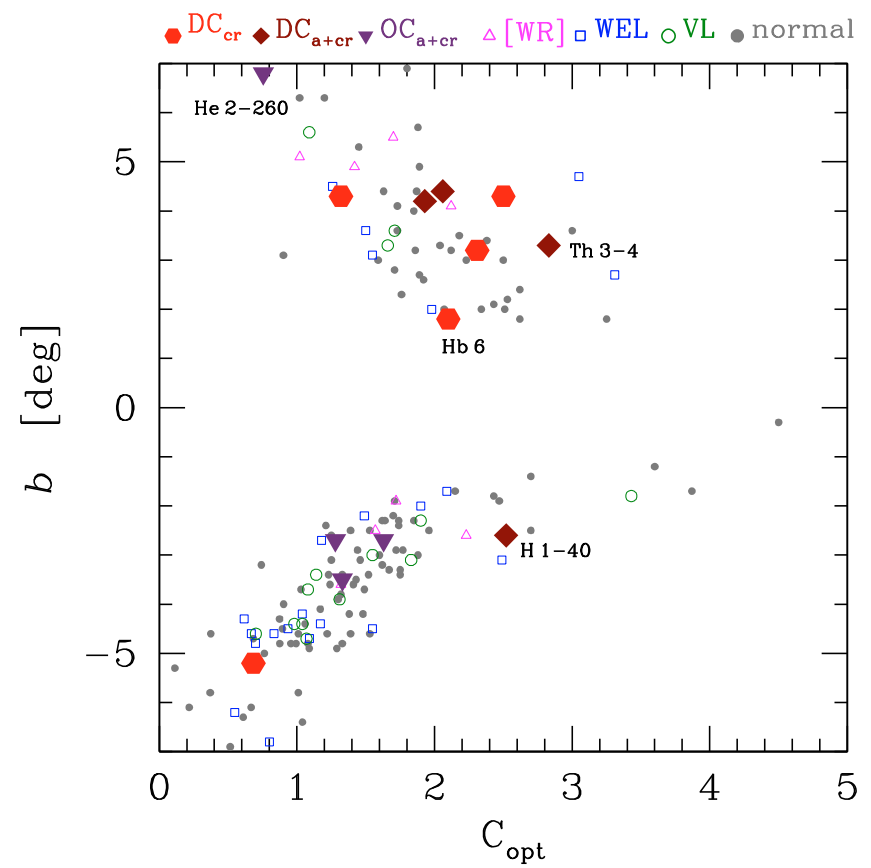

Fig. 3. Locations of investigated $\mathrm{PNe}$ in the Galactic latitude coordinate versus logarithmic extinction at $\mathrm{H} \beta$ parameter $C_{\text {opt }}$. The meaning of the symbols is the same as in Fig. 2.

of [WR] PNe in the bulge, comparable to the brightest of WEL $\mathrm{PNe}$ and usually brighter than VL PNe. The diameters of $\mathrm{DC}_{\mathrm{cr}}$ and $\mathrm{DC}_{\mathrm{a}+\mathrm{cr}}$ PNe range from about $1^{\prime \prime}$ to $5^{\prime \prime}$, suggesting that they are young or the nebulae are expanding slowly. However, expansion velocities are known for most of these objects (see Col. 7 of Table 2) and seem normal with a typical value of $20 \mathrm{~km} \mathrm{~s}^{-1}$. Using the data collected in Gesicki \& Zijlstra (2007), one can check that for the remaining GBPNe, normal, [WR], WEL and VL PNe combined (37 entries in their Table 3), the expansion 


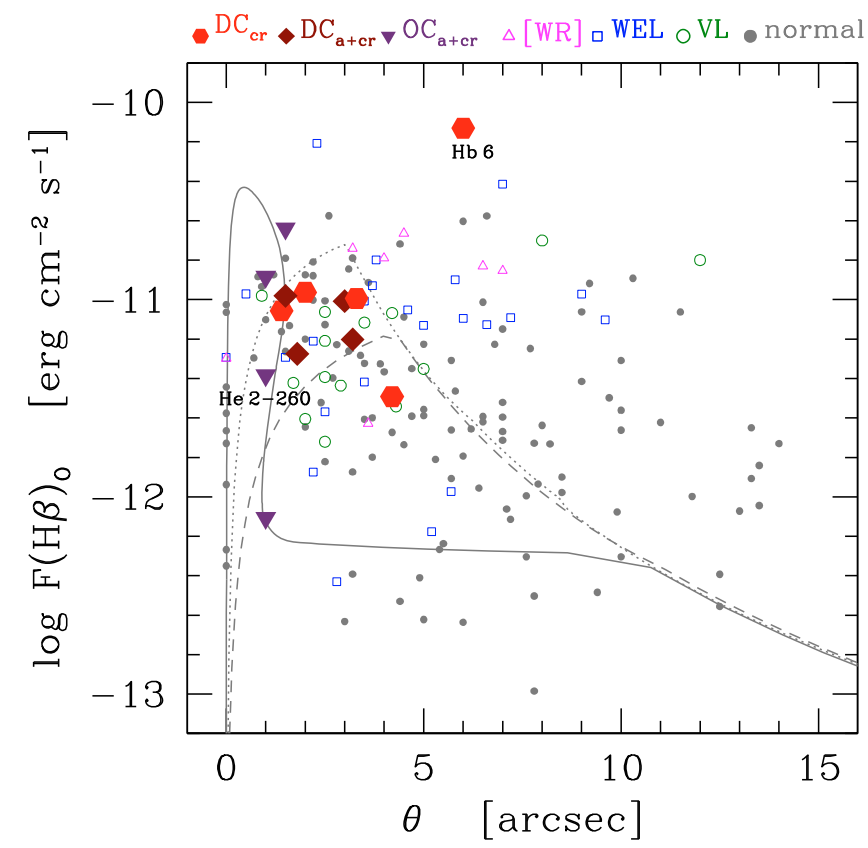

Fig. 4. The relation between apparent diameter and reddening-corrected $\mathrm{H} \beta$ flux for Galactic bulge PNe. The lines present model calculations for CSs of $0.57 M_{\odot}$ (dashed lines), $0.60 M_{\odot}$ (dotted), and $0.64 M_{\odot}$ (solid), adopting the total nebular mass of $0.20 M_{\odot}$. The meaning of the symbols is the same as in Fig. 2.

velocities have a rather flat distribution from 10 to $32 \mathrm{~km} \mathrm{~s}^{-1}$ with a median value of $V_{\exp }=22 \mathrm{~km} \mathrm{~s}^{-1}$.

The diameters of $\mathrm{OC}_{\mathrm{a}+\mathrm{cr}} \mathrm{PNe}$ are typically only $1^{\prime \prime}$, which are the smallest among known GBPNe. However there are indications, that their expansion velocities may be smaller than average as they range from $10 \mathrm{~km} \mathrm{~s}^{-1}$ to only $17 \mathrm{~km} \mathrm{~s}^{-1}$.

Assuming a simple model of constant nebular expansion that starts when the object leaves the AGB and knowing the angular diameter, distance, and $V_{\text {exp }}$, it is possible to derive the kinematic ages $t_{\text {kin }}$ of PNe. They are listed in Col. 7 of Table 2 and range from 500 to 3800 years. For comparison, the median age of GBPNe is 2900 years using data collected in Gesicki \& Zijlstra $(2007)^{6}$. This means that the objects analyzed here may not be extremely young but clearly belong to the younger PNe in the Galactic bulge. This is unsurprising taking into account the possible selection effects when choosing objects for Spitzer observations, as described in Sect. 2.

In Fig. 5, we present the distributions of electron densities derived from [SII] 6717/31 $\AA$ line ratio for the different groups of GBPNe. The values of $n_{\mathrm{e}}$ for individual objects can be found in Col. 5 of Table 2. Electron density is another independent parameter related to the age of the object. Surprisingly, as can be seen in Fig. 5, objects analyzed in this paper are among the densest of PNe in the Galactic bulge region. In particular, $\mathrm{OC}_{\mathrm{a}+\mathrm{cr}}$ and $\mathrm{DC}_{\mathrm{a}+\mathrm{cr}} \mathrm{PNe}$, i.e., the objects with the $10 \mu \mathrm{m}$ emission feature are much denser than the median $\log n_{\mathrm{e}}=3.14$ of normal GBPNe. The fact that they are also denser than the 75 percentile value of normal PNe implies that the distributions are truly different. The $\mathrm{OC}_{\mathrm{a}+\mathrm{cr}}$ and $\mathrm{DC}_{\mathrm{a}+\mathrm{cr}}$ are also considerably denser than the PNe with emission-line CSs.

\footnotetext{
6 This median age of GBPNe may be underestimated. There is a possibility of important selection effects in the sample of Gesicki \& Zijlstra (2007) that seems strongly biased towards PNe with small angular diameters as the median $\theta$ is $3{ }^{\prime \prime} 5$ in their sample compared with $5^{\prime \prime} .4$ of our normal PNe.
}

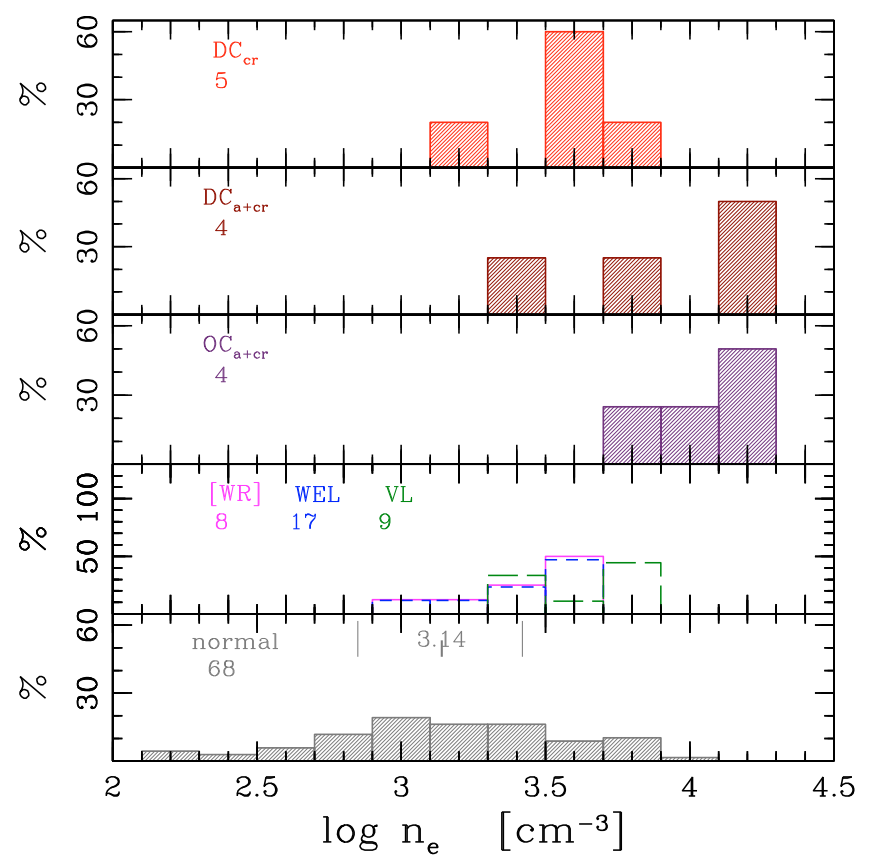

Fig. 5. Distributions of electron densities for the different groups of Galactic bulge PNe. For the normal PNe (bottom) the median value along with the 25 and 75 percentiles are marked with three short vertical lines above the histogram. Total numbers of objects included are shown in the left-hand parts of the panels below sample names.

\subsection{Evolutionary status}

The evolutionary state of the central star of the planetary nebula is best described by its temperature. In Col. 10 of Table 2, we present the stellar temperatures estimated with the Zanstra hydrogen method. Unfortunately, the data necessary to derive temperatures are not available for many $\mathrm{DC}_{\mathrm{cr}}$ and $\mathrm{DC}_{\mathrm{a}+\mathrm{cr}}$ objects and it is then difficult to compare them with the temperatures of the other GBPNe.

However, the most characteristic features of the planetary nebula spectrum are emission lines of many different atoms and their ions at different levels of excitation. As the temperature of the CS increases, the lines of increasingly higher ionization stages become observable. We take advantage of this property to at least qualitatively investigate the evolutionary stage of all the objects, including those with unknown CS temperature. For this purpose we divide them into three excitation classes: i) those characterized by nebulae in which most of the oxygen atoms remain in the form of $\mathrm{O}^{+}$ions i.e., $\mathrm{O}^{++} /\left(\mathrm{O}^{+}+\mathrm{O}^{++}\right)<$ 0.30 (for the coolest CSs); ii) those with most of their nebular oxygen in the form of $\mathrm{O}^{++}$but no or very little $\mathrm{He}^{++}$ions present i.e., $\mathrm{O}^{++} /\left(\mathrm{O}^{+}+\mathrm{O}^{++}\right)>0.30$ and $\mathrm{He}^{++} /\left(\mathrm{He}^{+}+\mathrm{He}^{++}\right)<$ 0.03 (for the intermediate temperature CSs); iii) the remaining objects with considerable amounts of helium $\mathrm{He}^{++}$ions i.e., $\mathrm{He}^{++} /\left(\mathrm{He}^{+}+\mathrm{He}^{++}\right)>0.03$ (for the hottest CSs). Using the three excitation classes so defined, we checked separately for each group of PNe discussed in this work the distributions of $\mathrm{H} \beta$ surface brightness. The parameters $\mathrm{O}^{++} /\left(\mathrm{O}^{+}+\mathrm{O}^{++}\right)$and $\mathrm{He}^{++} /\left(\mathrm{He}^{+}+\mathrm{He}^{++}\right)$for individual $\mathrm{DC}_{\mathrm{cr}}, \mathrm{DC}_{\mathrm{a}+\mathrm{cr}}$, and $\mathrm{OC}_{\mathrm{a}+\mathrm{cr}} \mathrm{PNe}$ are given in Cols. 4 and 5 of Table 2 and the values of $\mathrm{S}_{\mathrm{H} \beta}$ in Col. 9 of that table.

The $\mathrm{S}_{\mathrm{H} \beta}$ parameter constitutes a good measure of the evolutionary advancement of the nebula and changes by a few orders of magnitude between the formation of the observable nebula and the moment it disperses into the interstellar medium. In 

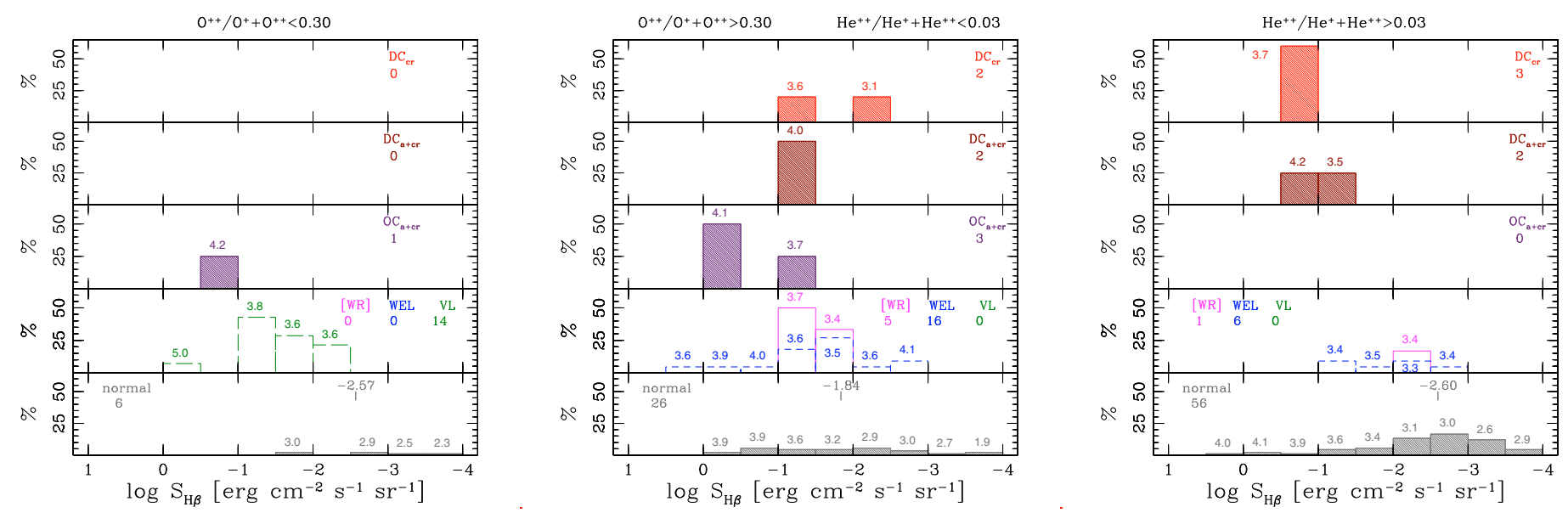

Fig. 6. The distribution of $\mathrm{S}_{\mathrm{H} \beta}$ surface brightness for different types of GBPNe. Separate histograms have been constructed for objects with different ionization classes as described at the top of the panels. Above each bar the logarithm of the mean electron density of the PNe comprising it is given $\left(\right.$ in $\mathrm{cm}^{-3}$ ).
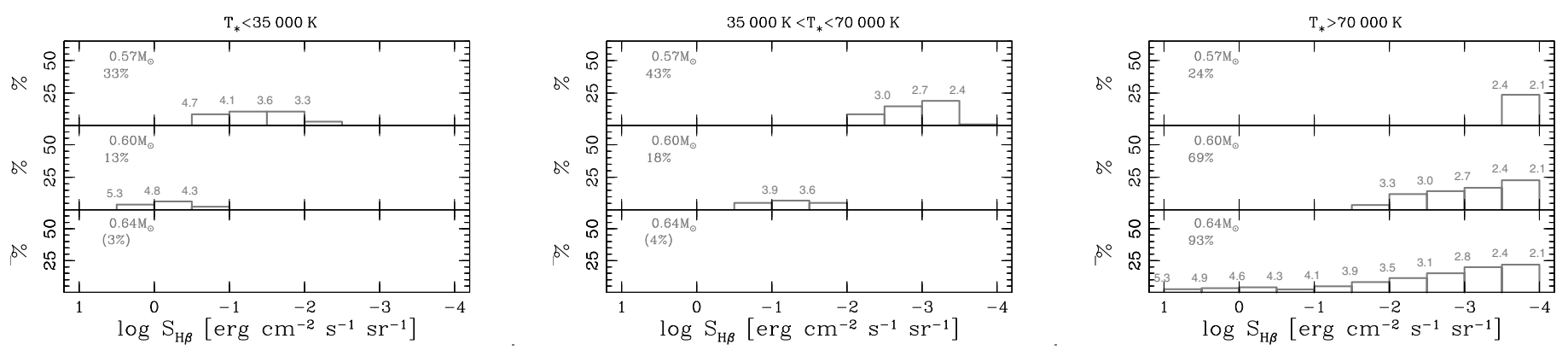

Fig. 7. Theoretical prediction of $\mathrm{S}_{\mathrm{H} \beta}$ distributions for PNe with total gas mass of $0.2 M_{\odot}$, parameter $\epsilon=0.75$, and expansion at $20 \mathrm{~km} \mathrm{~s}^{-1}$ for the CSs of $0.57,0.60$, and $0.64 M_{\odot}$. Separate histograms are presented for objects with CS temperature $T_{\star}<35 \mathrm{kK}$ (left panel), $35 \mathrm{kK}<T_{\star}<70 \mathrm{kK}$ (middle), and $T_{\star}>70 \mathrm{kK}($ right). The nebular electron densities are in addition plotted above the bar limits as expected from model calculations for PNe characterized by a given $\mathrm{S}_{\mathrm{H} \beta}$ value $\left(\log n_{\mathrm{e}}\right.$ in $\left.\mathrm{cm}^{-3}\right)$. The numbers under CS mass give the cumulative percentage of objects expected in the analyzed temperature domain (in parenthesis if $\mathrm{S}_{\mathrm{H} \beta}$ is expected larger than the presented range of values).

Fig. 6, we present the histograms of $\mathrm{S}_{\mathrm{H} \beta}$ for PNe with the coolest, intermediate, and hottest CSs in the left, middle, and right panels respectively. Above each histogram, the average nebular electron densities of the PNe that comprise the bars are overplotted.

In Fig. 7, we present for comparison the theoretical predictions for the histograms of $\mathrm{S}_{\mathrm{H} \beta}$. We used the same models as already presented in Sect. 4.2 and Fig. 4. We considered PNe with CSs of three different masses, 0.57, 0.60, and $0.64 M_{\odot}$ evolving according to the calculations of Blöcker (1995). The same simple model of surrounding nebula were assumed of uniformly filled sphere with total gas mass of $0.2 M_{\odot}$, filling factor $\epsilon=0.75$, and expanding at the constant velocity of $20 \mathrm{~km} \mathrm{~s}^{-1}$. To qualitatively reconstruct the ionization conditions applied above to the real observed $\mathrm{PNe}$, we divided the life of the model objects into three periods with CS temperature below $35 \mathrm{kK}$, between $35 \mathrm{kK}$ and $70 \mathrm{kK}$, and finally above $70 \mathrm{kK}$ (left, middle, and right panels in Fig. 7, respectively). The height of each bar is directly proportional to the period the object is characterized by the given $\mathrm{S}_{\mathrm{H} \beta}$. It is normalized by the total time nebula should be bright enough to be detected $(\log F(\mathrm{H} \beta)>-12.6)$ but not too extended and dispersed $(\Theta<14 \text { arcsec })^{7}$. The nebular electron densities expected from model calculations are also overplotted for the $\mathrm{S}_{\mathrm{H} \beta}$ starting and ending limit of each histogram bar.

Analyzing the distributions of $\mathrm{DC}_{\mathrm{cr}}$ and $\mathrm{DC}_{\mathrm{a}+\mathrm{cr}}$ in Fig. 6, we note that PNe of both groups seem to be at a similar stage

\footnotetext{
${ }^{7}$ Compare with the actual values for bulge PNe in Fig. 4.
}

of nebular evolution. Roughly half of their population seems to be associated with CSs of intermediate temperatures, while the other half has CSs that are already very hot. There are no $\mathrm{DC}_{\mathrm{cr}}$ nor $\mathrm{DC}_{\mathrm{a}+\mathrm{cr}} \mathrm{PNe}$ with cool CSs but the apparent deficit of objects with cool CSs seems to be a general property of GBPNe (with a clear exception of the VL PNe).

The $\mathrm{DC}_{\mathrm{cr}}$ and $\mathrm{DC}_{\mathrm{a}+\mathrm{cr}}$ with stellar temperatures in the range $35 \mathrm{kK}$ to $70 \mathrm{kK}$ are well reproduced by models with intermediate mass CSs (compare with predictions for $0.60 M_{\odot}$ star in Fig. 7). It is also important to note that their derived $\mathrm{S}_{\mathrm{H} \beta}$ have values typical of other GBPNe, including objects with emission-line CSs. Therefore, these $\mathrm{DC}_{\mathrm{cr}}$ and $\mathrm{DC}_{\mathrm{a}+\mathrm{cr}} \mathrm{PNe}$ do not seem to be less evolutionary advanced than [WR] or WEL PNe and thus cannot be their predecessors ${ }^{8}$.

A different situation can be seen in the right panel of Fig. 6, since the $\mathrm{S}_{\mathrm{H} \beta}$ of $\mathrm{DC}_{\mathrm{cr}}$ and $\mathrm{DC}_{\mathrm{a}+\mathrm{cr}}$ are clearly larger than those of both normal PNe and of the few GBPNe with hot emission-line CSs. By comparing with the models in Fig. 7, it is apparent that $\mathrm{DC}_{\mathrm{cr}}$ and $\mathrm{DC}_{\mathrm{a}+\mathrm{cr}} \mathrm{PNe}$ with CS temperatures above $70 \mathrm{kK}$ originate in more massive AGB progenitors than the group discussed above. Analyzing the model distributions, it can also be deduced that they should have a considerable number of unrecognized ancestors among normal PNe with hot CSs. The evolutionary

8 The existence of such predecessors with undiscovered or not yet active strong stellar winds could be one of the explanations why Galactic bulge [WR] PNe have only intermediate [WC] class CSs. 

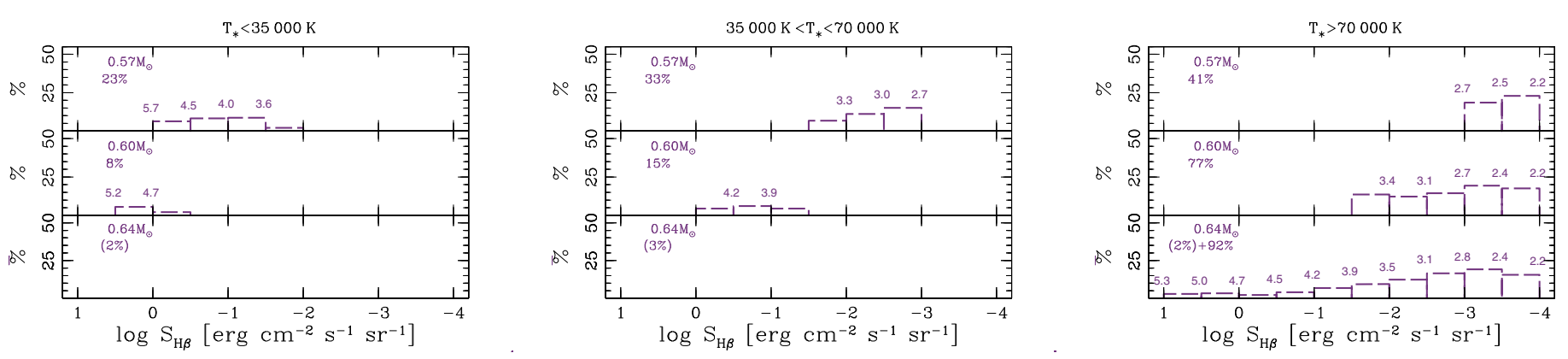

Fig. 8. Theoretical prediction of $\mathrm{S}_{\mathrm{H} \beta}$ distributions for PNe of $0.1 M_{\odot}$ and expanding at $12 \mathrm{~km} \mathrm{~s}^{-1}$ in the case of central stars of $0.57,0.60$, and $0.64 M_{\odot}$. The separate histograms are presented for objects with central star temperatures $T_{\star}<35 \mathrm{kK}$ (left panel), $35 \mathrm{kK}<T_{\star}<70 \mathrm{kK}($ middle) and $T_{\star}>70 \mathrm{kK}($ right $)$.

link to some PNe with emission-line CSs also cannot be excluded $^{9}$.

In summary, it seems that $\mathrm{DC}_{\mathrm{cr}}$ and $\mathrm{DC}_{\mathrm{a}+\mathrm{cr}} \mathrm{PNe}$ can be found among PNe with both massive and intermediate-mass CSs. As both groups are characterized by similar range of temperatures and $\mathrm{S}_{\mathrm{H} \beta}$ values but have clearly different electron densities (see Fig. 5), their evolutionary status cannot be identical. The details may be derived by future complete modeling. However, the higher electron densities of $\mathrm{DC}_{\mathrm{a}+\mathrm{cr}} \mathrm{PNe}$ could be explained if the distribution of gas was more clumpy in these PNe.

The $\mathrm{S}_{\mathrm{H} \beta}$ distributions of $\mathrm{OC}_{\mathrm{a}+\mathrm{cr}}$ PNe can be compared with the separate theoretical predictions in Fig. 8. Since the $\mathrm{OC}_{\mathrm{a}+\mathrm{cr}}$ PNe evolve more slowly, the expansion velocity of $12 \mathrm{~km} \mathrm{~s}^{-1}$ was assumed in these models (see in Sect. 4.4 and Table 2). In addition, a total nebular mass of $0.10 M_{\odot}$ was adopted so the models fit the small diameters of these PNe and do not exceed the measured electron densities. A qualitative agreement can be observed for $\mathrm{OC}_{\mathrm{a}+\mathrm{cr}}$ PNe with models of intermediate-mass CSs while models assuming lower-mass progenitors can be rejected. The only exception is He 2-260, an object with the coolest CS and a nebula with the fastest expansion among $\mathrm{OC}_{\mathrm{a}+\mathrm{cr}} \mathrm{PNe}$ that is probably located in the Galactic disk.

\subsection{Masses of nebular gas and dust}

Since the objects that we analyze were selected based on the appearance of their infrared spectra, it is important to learn about their dust content. The first important property is the dust-to-gas mass ratio $m_{\mathrm{d}} / m_{\mathrm{g}}$. It can be easily estimated from observables by adopting a simple model of the dusty nebula. We apply here the method from Stasińska \& Szczerba (1999) and recompute the parameters by adopting the new data on electron densities derived by Górny et al. (2009). In addition, dealing with Galactic bulge objects we can adopt the distance of $8.5 \mathrm{kpc}$ and derive absolute values of nebular ionized gas and dust masses. Our results are presented in Fig. 9 and in Cols. 11 and 12 of Table 2. For the Galactic disk PNe Hb 6 and He 2-260, the dust mass was calculated from the derived $m_{\mathrm{d}} / m_{\mathrm{g}}$ ratio by assuming $m_{\mathrm{g}}=0.2 M_{\odot}$.

As can be seen in Fig. 9, the dust content of $\mathrm{DC}_{\mathrm{cr}}$ and $\mathrm{DC}_{\mathrm{a}+\mathrm{cr}}$ $\mathrm{PNe}$ is usually similar to the typical value for normal GBPNe (median $\log m_{\mathrm{d}}=-3.30$, represented by horizontal short-dashed line in the plot). It can be recalled here that $\mathrm{DC}_{\mathrm{a}+\mathrm{cr}}$ can be suspected to have a considerable internal extinction (Sect. 4.1). However, since the dust content in these PNe is not exceptionally greater than in other objects, it could not explain excessive

\footnotetext{
9 The analysis is hampered by the Blöcker (1995) tracks being applicable directly only to $\mathrm{PNe}$ with $\mathrm{H}$-burning nuclei, i.e., not to [WR] type CSs.
}

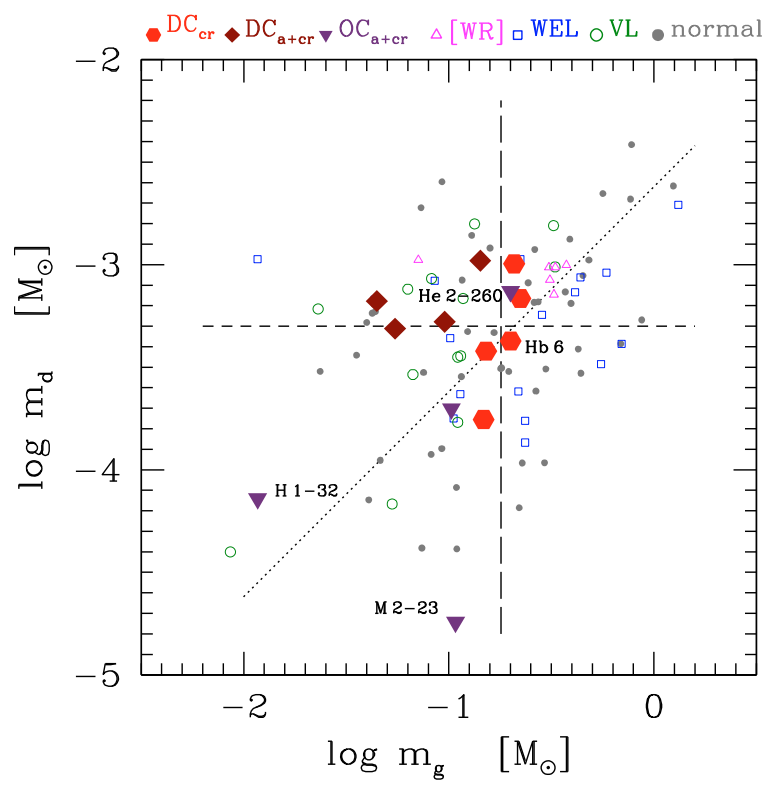

Fig. 9. The mass of the dust versus the mass of the nebular gas for Galactic bulge PNe. The meaning of the symbols is the same as in Fig. 2. The horizontal short-dashed and vertical long-dashed lines indicates, respectively, median $m_{\mathrm{d}}$ and $m_{\mathrm{g}}$ for normal bulge PNe (small dark grey symbols) and the dotted line indicate typical $m_{\mathrm{d}} / m_{\mathrm{g}}$ ratio of these PNe.

internal extinction. The reason for it would have to be in some specific properties of their dust that allow it to block more radiation than in other PNe.

As far as the dust-to-gas ratio of the PNe presented in Fig. 9 is concerned, the $\mathrm{DC}_{\mathrm{a}+\mathrm{cr}}$ represent the group with the highest ratio (see their location above the dotted line representing the median $m_{\mathrm{d}} / m_{\mathrm{g}}=2.47 \times 10^{-3}$ relation of normal $\left.\mathrm{PNe}\right)$. The $\mathrm{DC}_{\mathrm{cr}}$ are much closer to this line meaning their $m_{\mathrm{d}} / m_{\mathrm{g}}$ ratios have normal values. However, we recall that if some part of the nebula is still not ionized the value of $m_{\mathrm{d}} / m_{\mathrm{g}}$ that we derive may be overestimated. This is e.g., most probably true for the VL PNe that are in an extremely low ionization state (Górny et al. 2009). For $\mathrm{DC}_{\mathrm{a}+\mathrm{cr}} \mathrm{PNe}$, the derived ionized gas masses are all below the median mass of normal PNe in Fig. $9\left(m_{\mathrm{g}}=0.18 M_{\odot}\right.$, marked with vertical long-dashed line). This indicates that the $\mathrm{DC}_{\mathrm{a}+\mathrm{cr}} \mathrm{PNe}$ may also be only partially ionized, if their total nebular masses are similar to those of normal PNe.

As can be seen in Fig. 9, the mass of dust in individual $\mathrm{OC}_{\mathrm{a}+\mathrm{cr}}$ PNe may differ by more than an order of magnitude. For two of them M 2-23 and H 1-32, we derived very small values of $m_{\mathrm{d}}$ below $10^{-4} M_{\odot}$. On the other hand, except for M2-23, the $\mathrm{OC}_{\mathrm{a}+\mathrm{cr}}$ 
objects have $m_{\mathrm{d}} / m_{\mathrm{g}}$ ratio close to the value typical of normal PNe. If these nebulae were only partially ionized the true $m_{\mathrm{d}} / m_{\mathrm{g}}$ would be smaller than our estimate. Indeed, for $\mathrm{H}$ 1-32 the ionized gas mass is exceptionally low $m_{\mathrm{g}}=0.01 M_{\odot}$. For the other two bulge objects from the $\mathrm{OC}_{\mathrm{a}+\mathrm{cr}}$ group, the derived $m_{\mathrm{g}}$ is also only about one half of the typical mass of normal PNe.

\subsection{Dust temperature and infrared excess}

In Fig. 10, we present the dust color temperature $T_{\mathrm{d}}$ derived from the 25 and $60 \mu \mathrm{m}$ IRAS flux ratios versus infrared excess IRE defined as

$I R E=F_{\mathrm{IR}} / 22.3 F_{\mathrm{H} \beta}$,

where total infrared flux $F_{\text {IR }}$ has been estimated by computing the blackbody flux corresponding to the ratios of 25 to $60 \mu \mathrm{m}$ fluxes of IRAS. The values of both $T_{\mathrm{d}}$ and IRE were adopted from Stasińska \& Szczerba (1999). Figure 10 is found to be a good means of distinguishing all the different groups of GBPNe. There are two lines in this figure. The first represents the median IRE value for the normal GBPNe (dotted line) and the second the median $T_{\mathrm{d}}$ for normal PNe (dashed line). We note that almost all the nebulae that we analyze here, $\mathrm{DC}_{\mathrm{cr}}, \mathrm{DC}_{\mathrm{a}+\mathrm{cr}}$, and $\mathrm{OC}_{\mathrm{a}+\mathrm{cr}}$, as well as PNe with emission-line CSs ([WR], WEL, and VL) are located above the latter line, meaning they all have dust hotter than the typical $T_{\mathrm{d}}$ of normal PNe. The possible reason for this is that they are presumably at a relatively early or intermediate stage of PNe evolution when the dust is hotter (Stasińska \& Szczerba 1999). In Fig. 6 we can see that the majority of evolved objects (with $\log \mathrm{S}_{\mathrm{H} \beta}<-2$ ) are the normal PNe. In addition, selection effects may be important because strong dust emission (i.e., selection criterion for Spitzer/IRS targets) is more likely to be met in younger PNe with hotter dust when it is still relatively close to the CS.

In Fig. 10, it is quite convincing that the $\mathrm{OC}_{\mathrm{a}+\mathrm{cr}}$ belong to the objects with the hottest dust among bulge PNe. Apart from the above-mentioned relation with younger evolutionary age, other factors may be playing a role. The $T_{\mathrm{d}}$ can be higher in the case of low a $m_{\mathrm{d}} / m_{\mathrm{g}}$ ratio in the nebula and when amorphous silicate grains dominate the dust composition (see Fig. 7d, e of Stasińska \& Szczerba 1999). While there is only one $\mathrm{OC}_{\mathrm{a}+\mathrm{cr}}$ object with definitely very low $m_{\mathrm{d}} / m_{\mathrm{g}}$ (M 2-23, see in Sect. 4.4) the members of $\mathrm{OC}_{\mathrm{a}+\mathrm{cr}}$ are the only group of PNe analyzed here that show no clear signs of the $\mathrm{C}$-based dust and only evidence of the silicate dust grains are present in their spectra.

In their models, Stasińska \& Szczerba (1999) used the circumstellar silicates dominated by amorphous grains, whereas in spectra of $\mathrm{DC}_{\mathrm{cr}} \mathrm{PNe}$ and GBPNe with emission-line CSs there are clear signs of silicates in crystalline form only. It is not certain how different forms of silicate grains may influence the dust temperature. In Fig. 10, one can note that both IC 4776 - an unusual, unique WEL PN with $10 \mu \mathrm{m}$ amorphous feature and SwSt 1 - a [WR] type PN with a similar property belong to the hottest of nebulae of their respective types (compare also with Fig. 3 of Górny et al. 2001). On the other hand, the $\mathrm{DC}_{\mathrm{a}+\mathrm{cr}} \mathrm{PNe}$ that also exhibit amorphous silicates apparently have dust that is not convincingly hotter than for $\mathrm{DC}_{\mathrm{cr}} \mathrm{PNe}$ that do not show the $10 \mu \mathrm{m}$ feature.

The vertical dotted line in Fig. 10 indicates the median value of $\log$ IRE for normal PNe, which equals 0.63. This parameter and this particular value are important because it appears to separate the different groups of GBPNe. First, to the right of this line are $\mathrm{DC}_{\mathrm{a}+\mathrm{cr}} \mathrm{PNe}\left(\mathrm{OC}_{\mathrm{a}+\mathrm{cr}}\right.$ are discussed below) and the peculiar VL PNe with cool CSs. In the case of IRE, the model

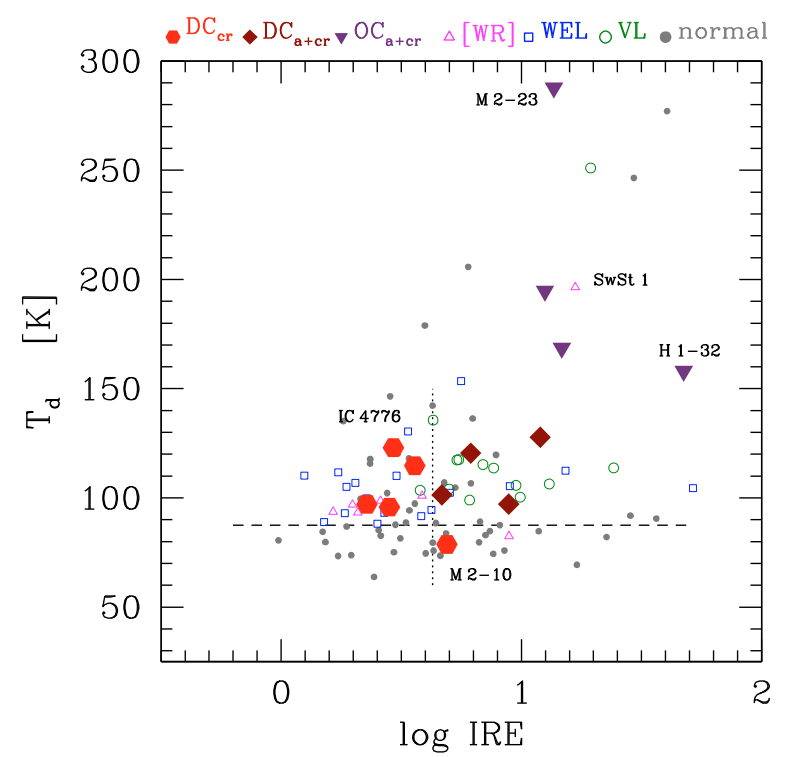

Fig. 10. The dust temperature versus the infrared excess parameter for Galactic bulge PNe. The meaning of the symbols is the same as in Fig. 2. Dashed and dotted lines indicate median values for normal bulge $\mathrm{PNe}$ (small black symbols).

calculations of Stasińska \& Szczerba (1999) show that, unlike $T_{\mathrm{d}}$ discussed above, this parameter is less dependent on the evolutionary state of the PN. However, it depends strongly on the $m_{\mathrm{d}} / m_{\mathrm{g}}$ of the nebula as it is expected to be greater in objects with larger dust content. This is confirmed by the locations of $\mathrm{DC}_{\mathrm{a}+\mathrm{cr}}$ and VL PNe in Fig. 9, which indicate higher than average ratios of the dust-to-gas mass in these PNe. On the other hand, the $\mathrm{DC}_{\mathrm{cr}}$ objects characterized by $m_{\mathrm{d}} / m_{\mathrm{g}}$ at the normal level (just like [WR] PNe, or even like WEL PNe with $m_{\mathrm{d}} / m_{\mathrm{g}}$ below normal) are located in Fig. 10 to the left of the dotted line with their IRE parameter being lower than average.

By analyzing the IRE parameter, we find the most puzzling case to be the $\mathrm{OC}_{\mathrm{a}+\mathrm{cr}}$ objects. The H1-32 nebula with its relatively large $m_{\mathrm{d}} / m_{\mathrm{g}}$ is also characterized by a large IRE in accordance with what we have just discussed. However, the other $\mathrm{OC}_{\mathrm{a}+\mathrm{cr}} \mathrm{PNe}$ with their (at most) moderate $m_{\mathrm{d}} / m_{\mathrm{g}}$ should have much lower IRE values. This is not the case. The most extreme example is M 2-23, which has the lowest $m_{\mathrm{d}} / m_{\mathrm{g}}$ ratio yet its IRE value is among the largest. By analyzing the model calculations of Stasińska \& Szczerba (1999), it seems that for OC $_{a+c r}$ the factor supporting larger IRE values may be their high density, e.g., due to their slower expansion. According to the models (Fig. 13a and 13c of Stasińska \& Szczerba 1999) IRE settles at an approximately constant level during an early phase of nebular evolution and becomes quite insensitive to other parameters. But if the nebular expansion is slow, as in the case of $\mathrm{OC}_{\mathrm{a}+\mathrm{cr}}$, the time before it occurs is extended and IRE can remain large for a longer time.

Finally, we should mention that the large IRE value of [WR]-type SwSt 1 nebula makes it again more similar to $\mathrm{OC}_{\mathrm{a}+\mathrm{cr}}$ or $\mathrm{DC}_{\mathrm{a}+\mathrm{cr}}$ than to bulge [WR] PNe in accordance with the large $m_{\mathrm{d}} / m_{\mathrm{g}}$ ratio of this object that can be inferred from Fig. 9.

\subsection{Chemical composition}

We present the chemical composition of the analyzed PNe derived from optical spectra with the classical empirical method. The values were taken from Górny et al. (2009) where the 
Table 3. Abundances of oxygen and nitrogen adopted from calculations by Górny et al. (2009) and compared to earlier published results.

\begin{tabular}{|c|c|c|c|}
\hline & \multicolumn{2}{|c|}{ This work } & \multirow{2}{*}{$\begin{array}{c}\text { Literature } \\
(\log \mathrm{O} / \mathrm{H}+12 ; \log \mathrm{N} / \mathrm{O})\end{array}$} \\
\hline & $\log \mathrm{O} / \mathrm{H}+12$ & $\log \mathrm{N} / \mathrm{O}$ & \\
\hline H 1-16 & 8.83 & -1.06 & $(9.09 ;-1.32)^{\mathrm{Sa}}$ \\
\hline $\mathrm{Hb} 6$ & 8.66 & -0.18 & $(8.71 ;-0.06)^{\mathrm{AK}}$ \\
\hline M 2-10 & 8.67 & -0.28 & $(8.78 ;-)^{\mathrm{G}} \quad(8.86 ;-0.49)^{\mathrm{R}} \quad(9.00 ;-0.55)^{K} \quad(8.90 ;-0.59)^{\mathrm{St}}$ \\
\hline H $1-50$ & 8.66 & -0.37 & $(8.65 ;-0.51)^{\mathrm{E}}$ \\
\hline H $1-20$ & 8.61 & -0.10 & $(8.97 ;-0.56)^{\mathrm{E}} \quad(8.45 ;-)^{\mathrm{G}} \quad(8.97 ;-0.23)^{\mathrm{R}} \quad(9.30 ;-0.67)^{\mathrm{Sa}} \quad(8.73 ;-0.44)^{\mathrm{K}}$ \\
\hline Th 3-4 & 8.22 & -0.11 & \\
\hline M 3-38 & 8.39 & 0.19 & $(8.37 ; 0.32)^{\mathrm{R}} \quad(8.79 ;-0.61)^{\mathrm{Sa}}(8.54 ;-0.22)^{\mathrm{K}} \quad(8.37 ;-)^{\mathrm{St}}$ \\
\hline M 3-8 & 8.38 & -0.26 & $(8.59 ;-0.42)^{\mathrm{E}}(9.02 ;-0.27)^{\mathrm{R}}(8.12 ;-0.27)^{\mathrm{C}}(8.15 ;-0.33)^{\mathrm{St}}$ \\
\hline H $1-40$ & 8.19 & -0.25 & $(9.09 ;-0.04)^{\mathrm{E}} \quad(8.23 ;-0.45)^{\mathrm{HS}} \quad(8.70 ;-0.62)^{\mathrm{R}} \quad(8.62 ; 0.02)^{\mathrm{St}}$ \\
\hline M 2-23 & 8.35 & -0.99 & $(8.48 ;-1.44)^{\mathrm{E}} \quad(8.67 ;-1.00)^{\mathrm{HS}} \quad(8.21 ;-0.13)^{\mathrm{St}} \quad(8.22 ;-0.82)^{\mathrm{R}} \quad(8.48 ; 0.20)^{\mathrm{K}}$ \\
\hline He 2-260 & 8.00 & -0.76 & \\
\hline H 1-32 & 8.59 & -1.21 & $(8.55 ;-0.23)^{\mathrm{St}}(8.12 ;-0.53)^{\mathrm{R}}$ \\
\hline H 1-35 & 8.65 & -1.19 & $(8.31 ;-0.92)^{\mathrm{R}} \quad(8.27 ;-0.89)^{\mathrm{K}}$ \\
\hline
\end{tabular}

Notes. Results by Cuisinier et al. (2000), Escudero et al. (2004), Górny et al. (2004) and Wang \& Liu (2007) are not presented as the line measurements from these papers were used by Górny et al. (2009).

(a) The values adopted are the averages of original calculations (see Table 4) by Górny et al. (2009) and from Stasińska et al. (1998).

References. AK - Aller \& Keyes (1987), C - Costa et al. (1996), E - Exter et al. (2004), G - Gutenkunst et al. (2008), R - Ratag et al. (1997), K - Köppen et al. (1991), Sa - Samland et al. (1992), St - Stasińska et al. (1998), HS - van Hoof \& van de Steene (1999).

applied method have been described in detail. One important difference of our study from analysis of Górny et al. (2009) is that we do not limit our discussion of $\mathrm{DC}_{\mathrm{cr}}, \mathrm{DC}_{\mathrm{a}+\mathrm{cr}}$, and $\mathrm{OC}_{\mathrm{a}+\mathrm{cr}}$ objects to parameters with errors smaller than 0.3 dex as in the case of some PNe their spectra do not allow for that quality.

The chemical elements in PNe are frequently divided into two groups. For one group of abundances it can be safely considered that they remain mostly unchanged during the previous evolution of the CS and therefore the values found in PNe represent the primordial abundances of the matter the progenitor star was born from. An example of such an element is oxygen, which is regarded as being mostly undisturbed since the object was born, at least in the case of PNe in metal-rich environments such as the Galactic bulge (see e.g., Chiappini et al. 2009, for detailed discussion). The abundances of other elements are however expected to be changed as the result of various physical processes. Their abundance ratios are modified by nuclear reactions and mixing that can bring some freshly synthesized matter to the stellar surface in so-called dredge-up processes. An example of such an element is nitrogen.

In Fig. 11, we plot the abundance ratios $\mathrm{O} / \mathrm{H}$ versus N/O for the different types of GBPNe. These data can also be found in Table 3, where there are compared to earlier results from the literature. As can be noted, the $\mathrm{DC}_{\mathrm{cr}}$ objects (except $\mathrm{H} 1-16$ ) have locations in this plane that are compatible with the majority of bulge PNe, including normal ones and those with [WR] or WEL type CSs. The locations of $\mathrm{DC}_{\mathrm{a}+\mathrm{cr}}$ are different as they show a substantial underabundance of oxygen related to both hydrogen and nitrogen. The $\mathrm{OC}_{\mathrm{a}+\mathrm{cr}} \mathrm{PNe}$ form another extreme with $\mathrm{N} / \mathrm{O}$ showing the lowest values among GBPNe, while $\mathrm{O} / \mathrm{H}$ seems normal. Interestingly, the unusual objects IC 4776 and SwSt 1 are located in the same region of the plot as $\mathrm{OC}_{\mathrm{a}+\mathrm{cr}} \mathrm{PNe}^{10}$.

Before considering the possible reasons of this behavior, one must first discuss whether the obtained oxygen abundances are reliable. As directly indicated in Fig. 11 by the errorbars, the formal errors of derived abundances are sometimes very large, especially for the $\mathrm{DC}_{\mathrm{a}+\mathrm{cr}} \mathrm{PNe}$. They were calculated by

\footnotetext{
10 We computed chemical abundances of SwSt 1 and IC 4776 using line intensities from Peña et al. (2001) and Exter et al. (2004).
}

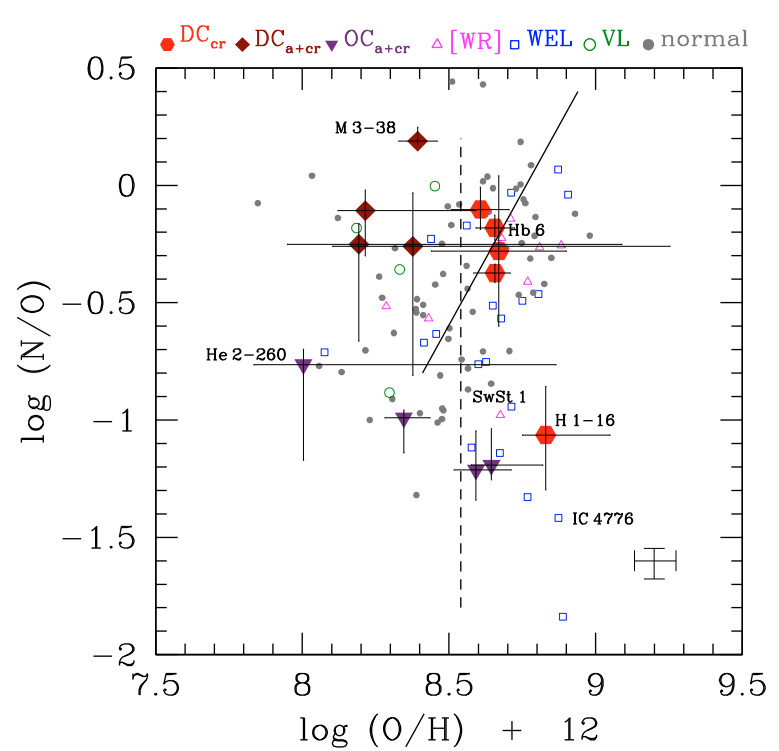

Fig. 11. The nebular abundance ratios $\mathrm{N} / \mathrm{O}$ versus $\mathrm{O} / \mathrm{H}$ for the different groups of GBPNe with meaning of the symbols the same as in Fig. 2. The solid line marks the relation between $\mathrm{O} / \mathrm{H}$ and N/O abundances (for $\mathrm{PNe}$ with $\log \mathrm{N} / \mathrm{O}>-0.8)$. The dashed vertical line marks median $\mathrm{O} / \mathrm{H}$ of normal PNe.

propagating the possible observational errors of measured spectral lines into computed parameters using the Monte Carlo method (for details see Górny et al. 2009). The typical errors for the other PNe presented in Fig. 11 are indicated with the errorbar cross in the bottom-right corner of the plot.

Despite the large individual errors, we observe that the $\mathrm{DC}_{\mathrm{a}+\mathrm{cr}} \mathrm{PNe}$ clearly have oxygen underabundances of more similar value than expected, because the random errors should produce a greater scatter in the distributions than observed. In Fig. 11, we mark with a solid thick line the relation between $\mathrm{O} / \mathrm{H}$ and N/O abundances for GBPNe with $\log \mathrm{N} / \mathrm{O}>-0.8$ and good quality data (rejecting only some clear outliers). Assuming that this holds for all PNe and that observed deviations from it are caused only by individual errors the probability of finding 
S. K. Górny et al.: New PNe with peculiar dust chemistry

Table 4. Chemical abundances for analyzed PNe and median values for bulge [WR], WEL, and normal PNe samples (from Górny et al. 2009).

\begin{tabular}{llrrccccc}
\hline \hline PN G & Name & He/H & N/H & O/H & Ne/H & S/H & $\mathrm{Ar} / \mathrm{H}$ & $\mathrm{Cl} / \mathrm{H}$ \\
\hline $000.1+04.3$ & H 1-16 & 11.05 & $5.83 \mathrm{E}-05$ & $6.76 \mathrm{E}-04$ & - & $6.08 \mathrm{E}-06$ & $2.94 \mathrm{E}-06$ & - \\
$007.2+01.8$ & Hb 6 & 11.09 & $2.99 \mathrm{E}-04$ & $4.53 \mathrm{E}-04$ & $1.47 \mathrm{E}-04$ & $9.84 \mathrm{E}-06$ & $3.72 \mathrm{E}-06$ & $1.20 \mathrm{E}-06$ \\
$354.2+04.3$ & M 2-10 & 11.15 & $2.99 \mathrm{E}-04$ & $2.73 \mathrm{E}-04$ & - & $7.93 \mathrm{E}-06$ & $3.58 \mathrm{E}-06$ & $7.69 \mathrm{E}-07$ \\
$358.7-05.2$ & H 1-50 & 11.07 & $1.92 \mathrm{E}-04$ & $4.54 \mathrm{E}-04$ & $1.31 \mathrm{E}-04$ & $7.36 \mathrm{E}-06$ & $2.35 \mathrm{E}-06$ & $1.63 \mathrm{E}-06$ \\
$358.9+03.2$ & H 1-20 & 11.16 & $3.20 \mathrm{E}-04$ & $4.05 \mathrm{E}-04$ & - & $7.51 \mathrm{E}-06$ & $3.98 \mathrm{E}-06$ & - \\
$354.5+03.3$ & Th 3-4 & 11.04 & $1.28 \mathrm{E}-04$ & $1.64 \mathrm{E}-04$ & $3.38 \mathrm{E}-05$ & $2.98 \mathrm{E}-06$ & $1.57 \mathrm{E}-06$ & - \\
$356.9+04.4$ & M 3-38 & 11.11 & $3.81 \mathrm{E}-04$ & $2.47 \mathrm{E}-04$ & $6.36 \mathrm{E}-05$ & $7.50 \mathrm{E}-06$ & $2.82 \mathrm{E}-06$ & $8.25 \mathrm{E}-07$ \\
$358.2+04.2$ & M 3-8 & 11.13 & $1.31 \mathrm{E}-04$ & $2.38 \mathrm{E}-04$ & - & $3.47 \mathrm{E}-06$ & $2.53 \mathrm{E}-06$ & - \\
$359.7-02.6$ & H 1-40 & 11.05 & $8.74 \mathrm{E}-05$ & $1.56 \mathrm{E}-04$ & - & $3.01 \mathrm{E}-06$ & $1.17 \mathrm{E}-06$ & - \\
$002.2-02.7$ & M 2-23 & 11.05 & $2.27 \mathrm{E}-05$ & $2.22 \mathrm{E}-04$ & $3.46 \mathrm{E}-05$ & $3.56 \mathrm{E}-06$ & $8.63 \mathrm{E}-07$ & $1.05 \mathrm{E}-06$ \\
$008.2+06.8$ & He 2-260 & 9.96 & $1.74 \mathrm{E}-05$ & $1.01 \mathrm{E}-04$ & - & $1.67 \mathrm{E}-06$ & $2.14 \mathrm{E}-07$ & - \\
$355.6-02.7$ & H 1-32 & 11.05 & $2.39 \mathrm{E}-05$ & $3.91 \mathrm{E}-04$ & - & $4.36 \mathrm{E}-06$ & $1.79 \mathrm{E}-06$ & - \\
$355.7-03.5$ & H 1-35 & 11.06 & $2.84 \mathrm{E}-05$ & $4.42 \mathrm{E}-04$ & $5.80 \mathrm{E}-05$ & $6.28 \mathrm{E}-06$ & $1.93 \mathrm{E}-06$ & $1.31 \mathrm{E}-06$ \\
[WR] & & 11.12 & $2.63 \mathrm{E}-04$ & $4.79 \mathrm{E}-04$ & $5.89 \mathrm{E}-05$ & $1.26 \mathrm{E}-05$ & $4.07 \mathrm{E}-06$ & $2.34 \mathrm{E}-06$ \\
WEL & & 11.08 & $7.41 \mathrm{E}-05$ & $4.47 \mathrm{E}-04$ & $1.10 \mathrm{E}-04$ & $4.90 \mathrm{E}-06$ & $2.04 \mathrm{E}-06$ & $3.02 \mathrm{E}-06$ \\
normal & & 11.14 & $1.26 \mathrm{E}-04$ & $3.47 \mathrm{E}-04$ & $7.76 \mathrm{E}-05$ & $5.89 \mathrm{E}-06$ & $2.09 \mathrm{E}-06$ & $1.51 \mathrm{E}-06$ \\
\hline
\end{tabular}

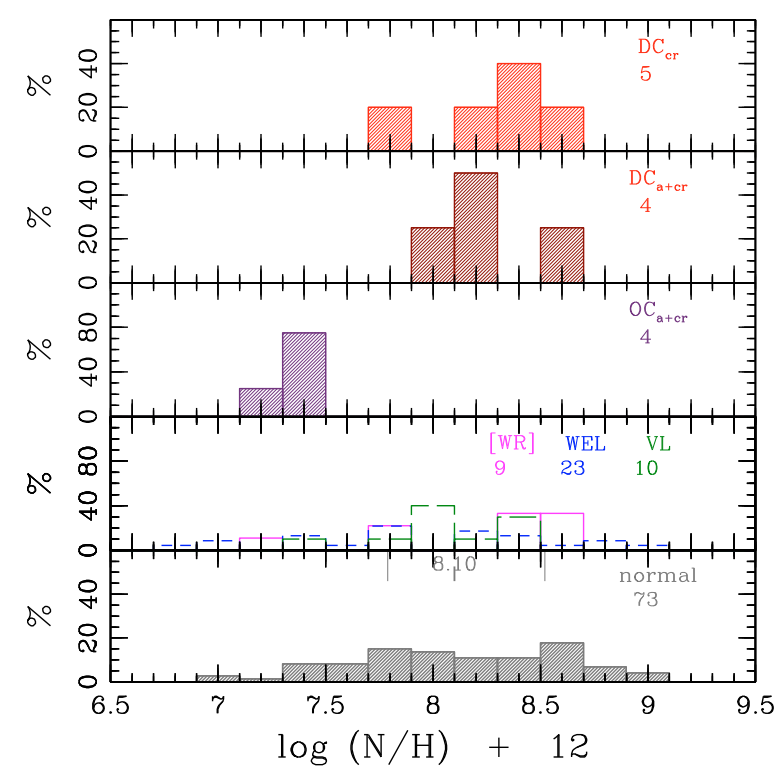

Fig. 12. Distributions of $\mathrm{N} / \mathrm{H}$ abundance ratio for the different groups of Galactic bulge PNe. The same notations apply as in Fig. 5.

all four $\mathrm{DC}_{\mathrm{a}+\mathrm{cr}}$ at their present locations in Fig. 11 can be evaluated as being smaller than $1 \%$. This hypothesis can therefore be safely rejected meaning that the $\mathrm{O} / \mathrm{H}$ vs. $\mathrm{N} / \mathrm{O}$ relation for $\mathrm{DC}_{\mathrm{a}+\mathrm{cr}}$ is truly significantly different from that of other GBPNe. Also using the Kolmogorov-Smirnoff 2D nonparametric test, the hypothesis that the difference between $\mathrm{DC}_{\mathrm{a}+\mathrm{cr}}$ and normal $\mathrm{PNe}$ in the Fig. 11 is statistically meaningful is confirmed at about $99 \%$ confidence level. That for the $\mathrm{DC}_{\mathrm{a}+\mathrm{cr}}$ object with the smallest errors (M3-38) we infer the same effect of oxygen underabundance $^{11}$ is also very important.

Columns 3 to 9 of Table 4 list the abundances of nitrogen and other elements for PNe investigated here and the median values for normal, [WR], and WEL GBPNe. In Fig. 12, we present the histograms of the $\mathrm{N} / \mathrm{H}$ ratio for the different types of GBPNe. Comparing the $\mathrm{DC}_{\mathrm{a}+\mathrm{cr}} \mathrm{PNe}$ with normal PNe, one can see no enhancement of nitrogen as the median values are almost the same. In the case of neon, sulphur, and possibly chlorine there

\footnotetext{
11 In fact, for M 3-38 we have two independent, high quality spectra that provide very similar results.
}

seems to be no depletion of these elements relative to oxygen (see Fig. 13). This means that whatever the reason for the lower oxygen abundance in $\mathrm{DC}_{\mathrm{a}+\mathrm{cr}} \mathrm{PNe}$ a similar decrease in the abundances of these three elements follows. Only in the case of argon is there possibly a difference at the level of $0.1 \mathrm{dex}$ in the median abundance ratio of $\mathrm{Ar} / \mathrm{O}$ between $\mathrm{DC}_{\mathrm{a}+\mathrm{cr}}$ and normal $\mathrm{PNe}$.

By analyzing the histograms of $\mathrm{DC}_{\mathrm{cr}}$ objects in Figs. 12 and 13 there is no indication of any important difference in chemical abundances between this group and normal $\mathrm{PNe}$.

The situation of $\mathrm{OC}_{\mathrm{a}+\mathrm{cr}} \mathrm{PNe}$ is different. As can be deduced from the histogram in Fig. 12, their underabundance of N/O is caused directly by the considerably lower number of nitrogen atoms. By analyzing the data for other elements in Fig. 13, we also find that the abundances of neon and argon seem lower than the average levels in normal PNe. On the other hand, no difference is noticeable for the abundances of chlorine and sulphur.

\section{Discussion}

There has been a long accepted opinion that post-AGB objects can belong to only one of the two groups: either those characterized by an oxygen-rich environment or those surrounded by carbon-dominated matter. However, soon after the first observations of Galactic PNe by ISO satellite were released it become clear that objects exist, that is [WR] PNe with late-type CSs, that simultaneously contain both carbon-based and oxygenbased dust. A number of hypotheses have been invoked to explain this phenomenon (see the review in Perea-Calderón et al. 2009). However, the dual-dust chemistry phenomenon remained to be viewed as a rare event among PNe that happens to only some objects. Furthermore, these objects were already acknowledged to be very peculiar because of the hydrogen-deficient composition of the CSs and strong WR-type stellar winds.

The Spitzer observations of GBPNe (Gutenkunst et al. 2008; Perea-Calderón et al. 2009) yielded a number of discoveries. The first surprise was a higher than expected percentage of PNe with dual-dust chemistry, which as we discussed in the Introduction might have been partly caused by the lower sensitivity of ISO. Gutenkunst et al. (2008) argued that dual-dust chemistry may be related to the binary evolution and the existence of circumbinary disks. They attributed the observed rate to a higher proportion of [WR] PNe in the Galactic bulge, which has not been actually proven (Górny et al. 2009), the Gutenkunst et al. (2008) sample containing only one such object. 

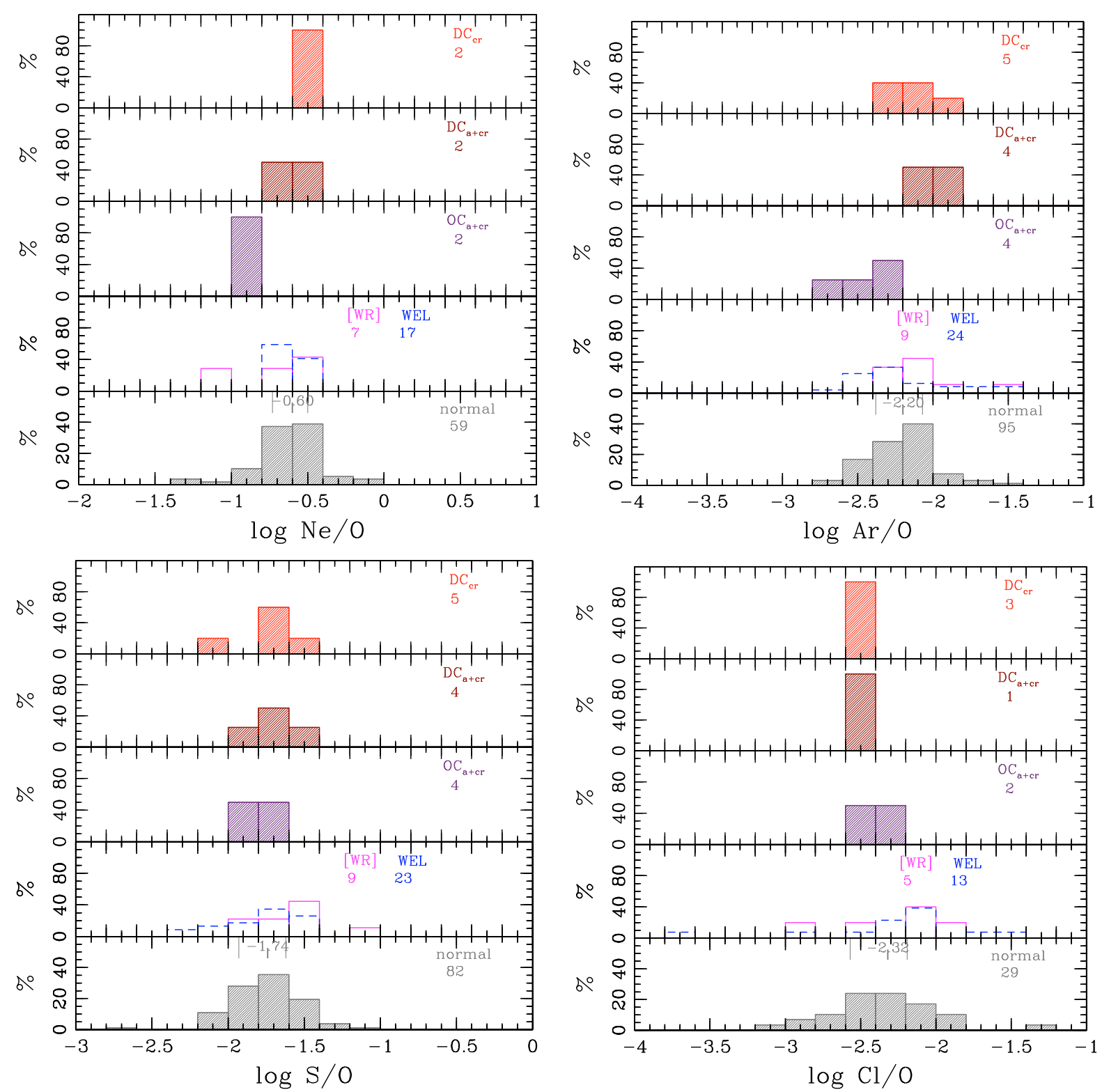

Fig. 13. Distributions of abundance ratios for different groups of Galactic bulge PNe. The same notations apply as in Fig. 5. Total number of objects included are given below sample names.

Analyzing their own sample of GBPNe Perea-Calderón et al. (2009) noted some other important results. The first was that dual-dust chemistry phenomenon is present not only in PNe surrounding late type [WC] CSs but in all [WR] $\mathrm{PNe}$ and also in all other PNe with emission-line CSs (WEL, VL) as well as many PNe without emission-line CSs. Perea-Calderón et al. (2009) also found that the percentage of PNe with C-rich material is confusingly high in the Galactic bulge since there is a well known deficit of C-rich AGB stars in that environment. We note that all GBPNe that contain PAHs are dual-dust chemistry sources.

Perea-Calderón et al. (2009) noted that the population of Orich AGB stars in the Galactic bulge, with the exception of obscured OH/IR stars (Vanhollebeke 2007), do not show any indication of crystalline silicates in their spectra. Thus the high detection rate of dual-dust chemistry found in $\mathrm{PNe}$ cannot be explained by long-lived O-rich (primordial or circumbinary) disks. That most of GBPNe cannot originate in the binary systems is indicated also by the results of Miszalski et al. (2009).
They reported that among 300 analyzed GBPNe only 21 (about $12-21 \%$ ) show the signatures of periodic variability in the OGLE-III data, which may be interpreted as being caused by close binarity.

The low-mass O-rich AGB stars in the Galactic bulge cannot bring enough carbon into the envelope to produce C-rich AGB stars since in the high-metallicity environment the efficiency of their third dredge-up is very limited. Therefore, Perea-Calderón et al. (2009) proposed that the most plausible scenario for creating C-rich AGB stars seems to be a final thermal pulse on the AGB (or just after), which would produce an enhanced mass loss, capable of removing/mixing (sometimes completely) the remaining $\mathrm{H}$-rich envelope and exposing the $\mathrm{C}$-rich layers. It would also generate shocks responsible for silicate crystallization in the ejected circumstellar shell.

For [WR] PNe there are many arguments that the change in the composition of the CS occurs predominantly on the AGB or soon after (Górny \& Tylenda 2000). Since dual-dust chemistry in the Galactic disk PNe had been revealed in [WR]-type objects 
it was natural to expect that a successful scenario should explain simultaneously both the unusual composition of [WR]-type CSs and the dual-dust chemistry of their nebulae (Górny 2008). The final thermal pulse at the end of the AGB suggested by Perea-Calderón et al. (2009) as a most plausible theory meets this expectation. However, it is unclear why dual-dust chemistry is so widely observed among GBPNe and restricted to only some Galactic disk objects. Perea-Calderón et al. (2009) pointed out that metallicity may be a possible explanation since the metallicities of GBPNe are on average higher than those in the Milky Way disk.

The properties of GBPNe with emission-line CSs of various types were investigated by Górny et al. (2009). In the present paper, we have therefore concentrated on those PNe that do not contain emission-line CSs. After inspecting the Spitzer spectra, we found that they can be divided into three ${ }^{12}$ clearly separate groups $\mathrm{DC}_{\mathrm{cr}}, \mathrm{DC}_{\mathrm{a}+\mathrm{cr}}$, and $\mathrm{OC}_{\mathrm{a}+\mathrm{cr}}$. Objects of the first group, $\mathrm{DC}_{\mathrm{cr}}$, have simultaneously both carbon-based dust (PAHs) and oxygen-based dust (crystalline silicates). In the second group, $\mathrm{DC}_{\mathrm{a}+\mathrm{cr}}$, there are both PAHs and crystalline silicates, but in addition there are also amorphous silicates. In the last group, $\mathrm{OC}_{\mathrm{a}+\mathrm{cr}}$, there is only oxygen-rich dust but in both crystalline and amorphous forms. Our primary discovery is that this classification based on the dust features in the infrared spectra is reflected in some other, more general properties of PNe.

The $\mathrm{DC}_{\mathrm{cr}} \mathrm{PNe}$ have infrared spectra that most closely resemble those of PNe with emission-line CSs. One can note the similar signatures of crystalline silicates at $23.5,27.5$, and $33.8 \mu \mathrm{m}$ and of PAHs at 6.2, “7.7", 8.6, and $11.3 \mu \mathrm{m}$. At the same time, the chemical composition of $\mathrm{DC}_{\mathrm{cr}}$ nebulae cannot be distinguished from that of the WEL objects and in general from the majority of GBPNe. In principle, one could consider whether $\mathrm{DC}_{\mathrm{cr}}$ are not an earlier evolutionary phase of WEL PNe when stellar emission lines are not yet visible but our analysis of their evolutionary state do not give strong support for such possibility. It cannot however be completely ruled out that for some $\mathrm{DC}_{\mathrm{cr}}$ $\mathrm{PNe}$, stellar emission-lines have not yet been discovered because of the quality of the spectra.

For the possible links between $\mathrm{DC}_{\mathrm{cr}}$ and [WR] PNe, it has to be noted that the latter objects are definitely brighter (see Fig. 4) and have more massive nebulae (Fig. 6). Both differences could be explained if $\mathrm{DC}_{\mathrm{cr}} \mathrm{PNe}$ are less evolutionary advanced. But in that case, it is hard to accept that relatively strong stellar emission-lines (expected spectral types should range from [WC11] to [WC7]) could remain unnoticed in the optical spectra. We can also add that large-scale turbulent motions are characteristic of PNe around [WR]-type CSs (Gesicki et al. 2006), however no information about them have so far been reported for any of $\mathrm{DC}_{\mathrm{cr}}$ PNe.

The objects belonging to $\mathrm{DC}_{\mathrm{a}+\mathrm{cr}}$ and $\mathrm{OC}_{\mathrm{a}+\mathrm{cr}}$ groups exhibit far more pronounced differences from the other GBPNe. The two groups are linked by showing evidence of amorphous silicates at $10 \mu \mathrm{m}$ and belonging to the densest PNe in the observed Galactic bulge population. However, the other observational results imply that the evolutionary status of $\mathrm{DC}_{\mathrm{a}+\mathrm{cr}}$ and $\mathrm{OC}_{\mathrm{a}+\mathrm{cr}}$ must be completely different.

The high densities of $\mathrm{DC}_{\mathrm{a}+\mathrm{cr}} \mathrm{PNe}$ can be regarded as a sign of the relatively short time that has passed since they left the AGB but it is more likely caused by their nebulae being more clumpy. $\mathrm{DC}_{\mathrm{a}+\mathrm{cr}}$ are also characterized by the considerable excess

12 There exist in the Galactic bulge also PNe with only signs of crystalline silicates but as explained in Sect. 2 we did not have enough data to discuss them as a separate class here. in extinction that could be attributed to some source of internal extinction. Finally, the nebular gas has a peculiar chemical composition with oxygen being underabundant relative to hydrogen, nitrogen, and possibly argon but preserving normal levels when compared to other elements. This seems difficult to interpret in the framework of the standard chemical evolution of PNe progenitors. If the $\mathrm{O} / \mathrm{H}$ in $\mathrm{DC}_{\mathrm{a}+\mathrm{cr}} \mathrm{PNe}$ were to represent the primordial oxygen abundance of the matter from which their CSs were formed, it would favor older objects born before the interstellar matter was enriched in metals. In that case however, the CSs of $\mathrm{DC}_{\mathrm{a}+\mathrm{cr}}$ PNe should be slowly evolving low mass objects and the N/O ratio should not be increased since the effective dredge-up of nitrogen occurs in higher mass AGB stars. In contrast, our analysis of the evolutionary status, indicate that $\mathrm{DC}_{\mathrm{a}+\mathrm{cr}}$ have intermediate and sometimes clearly higher mass CSs.

The low $\mathrm{O} / \mathrm{H}$ abundance accompanied by a higher than normal N/O ratio is possible if the ON cycle of nuclear reactions was active in the progenitor stars. In that case the $\mathrm{O} / \mathrm{H}$ ratio no longer reflects the primordial oxygen content. The ON cycle seems to work preferentially within more massive stars in low-metallicity environments. The examples are some $\mathrm{PNe}$ from LMC that show a clear anti-correlation between $\mathrm{O} / \mathrm{H}$ and N/O ratios (Leisy \& Dennefeld (2006), see also Chiappini et al. (2009) and the discussion therein). On the other hand, the ON cycle should have no effect on other elements. For the $\mathrm{DC}_{\mathrm{a}+\mathrm{cr}} \mathrm{PNe}$ discussed here, this is not the case as the abundances of neon, sulphur and chlorine closely follow the depletion of oxygen.

Finally, in $\mathrm{DC}_{\mathrm{a}+\mathrm{cr}}$ PNe some depletion of oxygen could be possible because it is being trapped in dust grains. The percentage of oxygen removed in this way from the gas to the dust may be metallicity dependent and is limited by the amount of silicon available. More could be depleted by means of ice growth but this would give rise to recognizable features in the infrared spectra. The depletion may simultaneously concern not only oxygen but also some other elements such as sulphur. However, it should have no effect on noble gas such as neon. As the Ne/O ratios of $\mathrm{DC}_{\mathrm{a}+\mathrm{cr}}$ nebulae are not enhanced, this hypothesis should also be ruled out.

The $\mathrm{OC}_{\mathrm{a}+\mathrm{cr}} \mathrm{PNe}$ are characterized by very small diameters and the highest densities but at the same time expand more slowly than other GBPNe. For this reason, their present evolutionary state indicates they should be related to intermediate mass CSs $\left(\approx 0.60 M_{\odot}\right)$. However, this is inconsistent with the very low metallicity of their surrounding nebulae as seen in the low N/O ratio derived for these objects as well as of neon and argon. In contrast, low metallicity argues for the low mass CSs originating in lower mass progenitors that were created before the Galactic bulge was effectively enriched in metals ${ }^{13}$.

The evolution of a low mass CS can be accelerated by high mass-loss. Kudritzki et al. (1997) detected very strong winds from $\mathrm{OC}_{\mathrm{a}+\mathrm{cr}}$ object $\mathrm{H}$ 1-35 (the final numerical value has not been given). On the other hand, He 2-260 has normal, low mass-loss at the level of $0.45 \times 10^{-7} M_{\odot} / y r$ (Hultzsch et al. 2007). In both cases the chemical composition of the CS is normal, i.e., they are not H-deficient.

The possible solution to the puzzle of the relatively fast evolution in the nebular phase and the low metallicity of $\mathrm{OC}_{\mathrm{a}+\mathrm{cr}}$ could be their origin not from single stars but from a binary system including a lower mass star. This star would not normally be observed in the PNe phase because its post-AGB evolution is too slow to ionize the ejected gas before it disperses. However, as a

\footnotetext{
13 In that case, the $\mathrm{O} / \mathrm{H}$ ratio may not represent the primordial composition, as is the case of PNe in the LMC - see Chiappini et al. (2009).
} 
result of the mass transfer from the companion at earlier evolutionary phases of the binary system (or even merging in extreme cases), the future CS could increase its mass and evolve more rapidly during its post-AGB phase. The PN would become visible, however, the nebular abundances of some elements possibly being characteristic of a lower mass (older) progenitor.

The evolution of the AGB star in the binary system can have other consequences. As argued by de Marco (2009) for nebulae created from common-envelope binaries, the abundances of e.g. nitrogen and carbon should be statistically lower that in "normal" PNe. This is because the interaction with the companion will cause the AGB star to terminate this phase of evolution earlier than of the single star. As a result, no effective dredge-up will take place associated with the thermal pulses at the tip of AGB. There will also be no final thermal pulse. The nebula M 2-23 was investigated by Miszalski et al. (2009) but no photometric variations were found. Nevertheless, the low N/O ratio is the characteristic feature of $\mathrm{OC}_{\mathrm{a}+\mathrm{cr}} \mathrm{PNe}$. We note also that the $\mathrm{OC}_{\mathrm{a}+\mathrm{cr}}$ are the only GBPNe that clearly do not have C-rich dust. At the same time, not all the surrounding silicate grains have been crystallized. All these results imply that the formation of $\mathrm{OC}_{\mathrm{a}+\mathrm{cr}} \mathrm{PNe}$ differs from that of the rest of the Galactic bulge population.

This leads us to the main conclusion of our work: there is clearly no unique road to the formation of PNe even in a uniform environment such as the Galactic bulge. This is noteworthy since the stars in the Galactic bulge that we are now able to observe in their PNe phase are expected to originate mostly from a single episode of star formation. Obviously, there are PNe in the Galactic bulge with very different CSs and different chemical compositions. They are also characterized by different properties of the dust as seen in $\mathrm{DC}_{\mathrm{cr}}, \mathrm{DC}_{\mathrm{a}+\mathrm{cr}}$, and $\mathrm{OC}_{\mathrm{a}+\mathrm{cr}}$ groups investigated in this paper. Nonetheless, the simultaneous presence of PAHs and crystalline silicates dominates in the GBPNe. Therefore, the scenario of final thermal pulse at the end of the AGB that changes both the stellar composition to C-rich and at the same time allows the crystallization of existing O-rich grains remains the most plausible possibility for the majority of GBPNe. However, it is not always effective or in different ways for different stars.

\section{Conclusions}

We have investigated PNe without emission-line central stars located towards the Galactic bulge that have peculiar infrared spectra acquired by Spitzer/IRS. Among these objects, we have found three separate groups divided according to their composition of dust grains:

- $\mathrm{DC}_{\mathrm{cr}}$ - dual-dust chemistry PNe with simultaneous presence of both carbon-based dust (PAHs) and oxygen-based dust (crystalline silicates);

- $\mathrm{DC}_{\mathrm{a}+\mathrm{cr}}$ - dual-dust chemistry PNe with simultaneous existence of PAHs and crystalline silicates as well as amorphous silicates;

- $\mathrm{OC}_{\mathrm{a}+\mathrm{cr}}-\mathrm{PNe}$ characterized by oxygen dust chemistry with only oxygen-rich grains in both crystalline and amorphous forms.

We have analyzed a wide range of different properties of these PNe. Our main results are:

- We confirm that dual-dust chemistry is a common phenomenon of PNe in the Galactic bulge and can occur in objects not related to emission-line central stars.
- The Properties of $\mathrm{DC}_{\mathrm{cr}}$ PNe do not distinguish them clearly from the majority of other PNe in the Galactic bulge. They have intermediate or higher-mass central stars. Their infrared spectra closely resemble those of PNe with emission-line nuclei. Some $\mathrm{DC}_{\mathrm{cr}}$ may be evolutionary related to the latter objects or may have undiscovered emission-line central stars.

- $\mathrm{DC}_{\mathrm{a}+\mathrm{cr}}$ objects belong to the densest PNe in the Galactic bulge. Their derived $m_{\mathrm{d}} / m_{\mathrm{g}}$ mass ratios and infrared excesses IRE are higher than average. There is a possibility of extensive internal extinction. $\mathrm{DC}_{\mathrm{a}+\mathrm{cr}}$ PNe have intermediate and higher-mass central stars. The chemical composition of nebular gas is peculiar as oxygen seems underabundant relative to hydrogen and nitrogen but not to other elements (except possibly argon). This composition of $\mathrm{DC}_{\mathrm{a}+\mathrm{cr}} \mathrm{PNe}$ cannot be explained in the standard picture of AGB star chemical evolution.

- The $\mathrm{OC}_{\mathrm{a}+\mathrm{cr}} \mathrm{PNe}$ are the only analyzed PNe not showing dual-dust chemistry. They have hottest dust temperature $T_{\mathrm{d}}$ and highest infrared excess IRE. $\mathrm{OC}_{\mathrm{a}+\mathrm{cr}}$ have also very small diameters and are among the densest PNe in the Galactic bulge. However, their expansion velocities are smaller than average and therefore their evolutionary status indicates that $\mathrm{OC}_{\mathrm{a}+\mathrm{cr}}$ can have intermediate-mass central stars. In contrast, the surrounding nebulae show low metallicity with an underabundance of nitrogen, neon, and argon. The domination of oxygen-based dust indicates in addition a low abundance of carbon. We argue that their properties are in qualitative agreement with scenarios of PNe formation not from single AGB stars but from binary systems.

Acknowledgements. We acknowledge support from the Faculty of the European Space Astronomy Centre (ESAC) and from the Comunidad de Madrid PRICIT project S-0505/ESP-0237 (ASTROCAM). R.Sz. and S.K.G. acknowledge support from grant N203 393334 of the Science and High Education Ministry of Poland. D.A.G.H. acknowledges support for this work provided by the Spanish Ministry of Science and Innovation (MICINN) under the 2008 Juan de La Cierva Program and under grant AYA-2007-64748.

\section{References}

Aller, L. H., \& Keyes, C. D. 1987, ApJS, 65, 405

Beaulieu, S. F., Freeman, K. C., Kalnajs, A. J., Saha, P., \& Zhao, H. 2000, AJ, 120,855

Blöcker, T. 1995, A\&A, 299, 755

Cerrigone, L., Hora, J. L., Umana, G., \& Trigilio, C. 2009, ApJ, 703, 585

Chiappini, C., Górny, S. K., Stasińska, G., \& Barbuy, B. 2009, A\&A, 494, 591

Cohen, M., Barlow, M. J., Sylvester, R. J., et al. 1999, ApJ, 513, L135

Cohen, M., Barlow, M. J., Liu, X.-W., \& Jones, A. F. 2002, MNRAS, 332, 879

Costa, R. D. D., Chiappini, C., Maciel, W. J., \& de Freitas Pacheco, J. A. 1996, A\&AS, 116, 249

Crowther, P. A. 2008, in Hydrogen-Deficient Stars, ed. A. Werner, \& T. Rauch, ASP Conf. Ser., 391, 83

Cuisinier, F., Maciel, W. J., Köppen, J., Acker, A., \& Stenholm, B. 2000, A\&A, 353,543

de Marco, O. 2009, PASP, 121, 316

Durand, S., Acker, A., \& Zijlstra, A. 1998, A\&AS, 132, 13

Escudero, A. V., \& Costa, R. D. D. 2001, A\&A, 380, 300

Escudero, A. V., Costa, R. D. D., \& Maciel, W. J. 2004, A\&A, 414, 211

Exter, K. M., Barlow, M. J., \& Walton, N. A. 2004, MNRAS, 349, 1291

García-Hernández, D. A., García-Lario, P., Plez, B., et al. 2007, A\&A, 462, 711

García-Lario, P., Manchado, A., Ulla, A., \& Manteiga, M. 1999, ApJ, 513, 941

Gesicki, K., \& Zijlstra, A. A. 2000, A\&A, 358, 1058

Gesicki, K., \& Zijlstra, A. A. 2007, A\&A, 467, L29

Gesicki, K., Zijlstra, A. A., Acker, A., et al. 2006, A\&A, 451, 925

Górny, S. K. 2008, in Hydrogen-Deficient Stars, ed. A. Werner \& T. Rauch, ASP Conf. Ser., 391, 165

Górny, S. K., \& Tylenda, R. 2000, A\&A, 362, 1008

Górny, S. K., Stasińska, G., Szczerba, R., \& Tylenda, R. 2001, A\&A, 377, 1007 Górny, S. K., Stasińska, G., Escudero, A. V., \& Costa, R. D. D. 2004, A\&A, 427, 231

Górny, S. K., Chiappini, C., Stasińska, G., \& Cuisinier, F. 2009, A\&A, 500, 1089 
Gutenkunst, S., Bernard-Salas, J., Pottasch, S. R., Sloan, G. C., \& Houck, J. R. 2008, ApJ, 680, 1206

Herwig, F. 2005, ARA\&A, 43, 435

Houck, J. R., Roellig, T. L., van Cleve, J., et al. 2004, ApJS, 154, 18

Hultzsch, P. J. N., Puls, J., Méndez, R. H., et al. 2007, A\&A, 467, 1253

Köppen, J., Acker, A., \& Stenholm, B. 1991, A\&A, 248, 197

Kudritzki, R. P., Mendez, R. H., Puls, J., \& McCarthy, J. K. 1997, in Planetary Nebulae, ed. H. J. Habing, \& H. J. G. L. M. Lamers, IAU Symp., 180, 64

Leisy, P., \& Dennefeld, M. 2006, A\&A, 456, 451

Miszalski, B., Acker, A., Moffat, A. F. J., Parker, Q. A., \& Udalski, A. 2009, A\&A, 496, 813

Molster, F. J., Waters, L. B. F. M., Tielens, A. G. G. M., \& Barlow, M. J. 2002, A\&A, 382, 184

Peña, M., Stasińska, G., \& Medina, S. 2001, A\&A, 367, 983

Perea-Calderón, J. V., García-Hernández, D. A., García-Lario, P., Szczerba, R., \& Bobrowsky, M. 2009, A\&A, 495, L5

Ratag, M. A., Pottasch, S. R., Dennefeld, M., \& Menzies, J. 1997, A\&AS, 126, 297

Richer, M. G., López, J. A., Pereyra, M., et al. 2008, ApJ, 689, 203

Robinson, G. J., Reay, N. K., \& Atherton, P. D. 1982, MNRAS, 199, 649

Samland, M., Koeppen, J., Acker, A., \& Stenholm, B. 1992, A\&A, 264, 184
Stanghellini, L., García-Lario, P., García-Hernández, D. A., et al. 2007, ApJ, 671,1669

Stasińska, G., \& Szczerba, R. 1999, A\&A, 352, 297

Stasińska, G., Richer, M. G., \& McCall, M. L. 1998, A\&A, 336, 667

Szczerba, R., Górny, S. K., Stasińska, G., Siódmiak, N., \& Tylenda, R. 2001, Ap\&SS, 275, 113

Szczerba, R., Stasińska, G., Siódmiak, N., \& Górny, S. K. 2003, in Exploiting the ISO Data Archive. Infrared Astronomy in the Internet Age, ed. C. Gry, S. Peschke, J. Matagne, P. Garcia-Lario, R. Lorente, \& A. Salama, ESA SP, 511,149

Tylenda, R., Acker, A., \& Stenholm, B. 1993, A\&AS, 102, 595

Uttenthaler, S., Hron, J., Lebzelter, T., et al. 2007, A\&A, 463, 251

van Hoof, P. A. M., \& van de Steene, G. C. 1999, MNRAS, 308, 623

Vanhollebeke, E. 2007, Ph.D. Thesis, Katholieke Universiteit Leuven, Leuven

Wang, W., \& Liu, X.-W. 2007, MNRAS, 381, 669

Waters, L. B. F. M., Beintema, D. A., Zijlstra, A. A., et al. 1998a, A\&A, 331, L61

Waters, L. B. F. M., Cami, J., de Jong, T., et al. 1998b, Nature, 391, 868

Werner, M. W., Roellig, T. L., Low, F. J., et al. 2004, ApJS, 154, 1

Zoccali, M., Renzini, A., Ortolani, S., et al. 2003, A\&A, 399, 931 